A N N A L E S Annales de Bretagne et des Pays de l'Ouest

\title{
Le commerce des Français à Cadix vu par les consuls de France (1763-1778)
}

\section{Olivier Le Gouic}

\section{(2) OpenEdition}

Journals

\section{Édition électronique}

URL : http://journals.openedition.org/abpo/1119

DOI : 10.4000/abpo. 1119

ISBN : 978-2-7535-1499-7

ISSN : 2108-6443

Éditeur

Presses universitaires de Rennes

Édition imprimée

Date de publication : 20 septembre 2005

Pagination : 71-104

ISBN : 978-2-7535-0201-7

ISSN : 0399-0826

\section{Référence électronique}

Olivier Le Gouic, "Le commerce des Français à Cadix vu par les consuls de France (1763-1778) »,

Annales de Bretagne et des Pays de l'Ouest [En ligne], 112-3 | 2005, mis en ligne le 20 septembre 2007, consulté le 21 avril 2019. URL : http://journals.openedition.org/abpo/1119 ; DOI : 10.4000/abpo.1119 


\title{
Le commerce des Français à Cadix vu par les consuls de France (1763-1778)
}

\author{
Olivier LE GouIC \\ Professeur certifié d'Histoire-Géographie \\ SOLITO - Université de Bretagne Sud
}

$\mathrm{Au}$ lendemain de la guerre de Sept Ans, Cadix est une place marchande majeure en Europe. Antonio Garcia-Baquero Gonzalez en fait avec raison le « trait d'union entre l'Europe commerciale et maritime et l'immense continent américain ${ }^{1}$ ». Depuis le transfert de la Casa de Contratacion en 1717, la population de la ville a considérablement augmenté, passant de 20000 habitants au début du $\mathrm{XVIII}^{\mathrm{e}}$ siècle à plus de 60000 à l'époque où nous nous situons. Cette croissance démographique spectaculaire doit beaucoup à l'immigration, et en particulier à l'arrivée de nombreux Français. Cadix est en effet devenue " le but de tout homme d'affaires qui désire faire fortune " et la communauté française voit ses effectifs doubler au cours du XVIII ${ }^{\mathrm{e}}$ siècle, au terme d'une " croissance soutenue ${ }^{2}$ ". Le recensement de 1764 évalue à 506 le nombre de négociants et de marchands étrangers installés dans le port andalou, dont 219 Français, soit près d'un quart des négociants français alors établis dans la péninsule ${ }^{3}$. À ces chefs de maisons, il faut ajouter les commis, caissiers, teneurs de livres et domestiques, ce qui porte à 624 le nombre de Français ${ }^{4}$, mais aussi les femmes et les enfants, sans oublier non plus une population de petits métiers difficilement repérable car très mobile, tant géographiquement que socialement. Retenons parmi eux les boulangers, perruquiers, tailleurs, cordonniers,

1. Garcia-Baquero Gonzalez, Antonio, Collado Villalta, Pedro, " Les Français à Cadix au XVIII ${ }^{\mathrm{e}}$ siècle : la colonie marchande ", Les Français en Espagne à l'époque moderne (XVI XVIII siècles), Paris, CNRS, 1990 p. 173.

2. Ibidem, p. 194.

3. ZYLBERBERG, Michel, Une si douce domination. Les milieux d'affaires français et l'Espagne vers 1780-1808, Paris, Comité pour l'Histoire économique et financière de la France, 1993, p. 81; sur un millier d'hommes d'affaires français implantés dans la péninsule en 1764, 219 sont installés à Cadix, soit dix fois plus qu'à Séville ou Bilbao, près de six fois plus qu'à Madrid et près de cinq fois plus qu'à Valence.

4. Ibidem, p. 121. 
porteurs d'eau, faiseurs de chaises, vendeurs d'huile et de charbon par les rues, garçons d'auberge, regratiers et autres gagne-petit évoqués par les députés de la Nation française en $1771^{5}$. En 1773, un autre padron recense 910 Français ${ }^{6}$. Ils représentent alors la moitié des étrangers installés dans la ville. Sur la base d'une liste de vecinos élaborée dans le cadre d'un recensement militaire, Antonio Garcia-Baquero réévalue à 1459 le nombre total de Français présents à Cadix en $1773^{7}$. Le gouverneur de Cadix avait d'ailleurs, au nom du Roi d'Espagne, demandé à cette occasion à tous les consuls étrangers de dresser un " matricule ou liste générale de tous les individus dont se compose chaque nation étrangère ", notamment pour bien veiller à exclure du tirage au sort prévu pour la levée de la milice " les naturels du pays qui se trouveront employés dans les maisons des négociants en gros espagnols ou étrangers en qualité de teneurs de livres de correspondance et des caisses ${ }^{8}$ ". En janvier 1777, le consul De Mongelas dresse à son tour une " liste des négociants, boutiquiers, artisans, domestiques et autres sujets du Roi établis et résidents présentement à Cadix ${ }^{9}$ ". Il s'agit cette fois " entr'autres choses de faire contribuer aux charges de la Nation tous les Français matriculés réputés négociants et de les répartir dans les différentes classes de la Nation en fonction de leurs besoins ${ }^{10}$ ". Cette liste comprend 885 noms et donne l'image d'une communauté que Didier Ozanam décrit comme étant divisée en trois catégories : " Au sommet, les grandes maisons de commerce et les négociants en gros; au dessous, les boutiquiers et autres marchands détailleurs; plus bas encore, le monde des manuels, artisans et petits métiers ${ }^{11}$."

Quoi qu'il en soit, avec certainement plus d'un millier de ressortissants, la colonie française constitue une composante importante de la société gaditane. Certains de ses membres sont totalement intégrés, et ce parfois depuis plusieurs générations quand leur famille a fait souche, concluant des alliances matrimoniales avec le négoce local ${ }^{12}$. Ce sont les domiciliados ou avecinados qu'il faut distinguer des transeuntes ou étrangers de passage, ceux qui, conformément à une déclaration royale de 1716, n'ont pas

5. Arch. nat., Aff. étr. BI 279, copie d'une lettre écrite par les députés de la Nation française à Cadiz au marquis d'Ossun, ambassadeur du Roi à Madrid (16 avril 1771), jointe à une lettre du consul De Puyabry du 23 avril 1771.

6. OzAnAm, Didier, « La colonie française de Cadix au XVIII ${ }^{\mathrm{e}}$ siècle d'après un document inédit de 1777 ", Mélange de la Casa de Velazquez (tome IV), Paris, éditions du Boccard, 1968, p. 263.

7. Sur la base d'un document conservé aux archives municipales de Cadix, section padrones 1006-7; Garcia-BaQuero, Antonio, Collado Villalta, Pedro, " Les Français à Cadix au XVIII ${ }^{\mathrm{e}}$ siècle... ", op. cit., p. 176.

8. Arch. nat., Aff. étr. BI 280, lettres de De Puyabry du 9 avril et du 25 juin 1773.

9. Arch. nat., Aff. étr. BI 283 f $^{\circ}$ 5, lettre de De Mongelas du 20 janvier 1777.

10. Arch. nat., Aff. étr. BI 283, lettre de De Mongelas du 10 janvier 1777.

11. OzanAm, Didier, "La colonie française de Cadix... ", op. cit., p. 265.

12. "Une minorité de cas " selon Antonio Garcia-Baquero Gonzalez et Pedro Collado Villalta qui donnent, en conclusion de leur article, une liste de neuf familles "intégrées par voie de naturalisation " et devenues " les artisans de la permanence parmi les commerçants français ", "Les Français à Cadix au XVIII ${ }^{\mathrm{e}}$ siècle ", op. cit., p. 194-195. 
encore " fait un séjour de dix ans avec établissement de maison " et qui, de ce fait, ne sont pas " attachés au pavillon et au Consulat de la Nation ${ }^{13}$ ".

D'après les procès verbaux des délibérations des assemblées de la Nation, il y avait à Cadix 63 sociétés françaises de commerce en gros en 1763, 84 en 1771, 70 en $1784^{14}$. Elles représentent l'élite des milieux d'affaires en Espagne à cette époque. On y retrouve des grands noms du négoce français comme Magon ${ }^{15}$, Le Fer ou Verduc de Saint-Malo, Solier de Marseille, Le Couteulx de Paris, Gilly et Fornier de Nîmes ${ }^{16}$, Delabat et Rey de Lyon... Tous sont attirés par la même chose, la possibilité de gagner de l'argent en approvisionnant la ville en denrées et marchandises de France - au premier plan desquelles le blé, les toiles, les étoffes - ou en s'immisçant dans le commerce des Indes. Le « commerce d'Espagne " est alors une composante essentielle du commerce extérieur français. Michel Zylberberg rappelle, dans son ouvrage sur les milieux d'affaires français en Espagne, qu'il n'est dépassé en valeur que par le commerce colonial. À partir des balances du commerce, il évalue le montant moyen annuel des échanges entre les deux royaumes à un peu plus de 69 millions de livres pour les années 1764,1765 et 1766 , et à près de 79 millions de livres par an pour les années 1775 à $1777^{17}$. À la fin de notre période, la valeur moyenne des exportations vers l'Espagne, qui avait pu égaler le double de la valeur des importations au lendemain du traité de Paris, était encore supérieure d'un quart à celle des importations. Au milieu des ballots qui s'amoncellent à chaque fois qu'un navire français décharge dans le port de Cadix, les toiles, en particulier celles qui proviennent de Bretagne, s'imposent comme la marchandise la plus prisée par le commerce gaditan, au point de composer à elles seules la moitié des cargaisons. Les produits textiles constituent alors près des trois quarts des arrivages en provenance des ports français. Après les toiles viennent les soieries, les lainages, les chapeaux, les denrées alimentaires, les chemises, les articles de mercerie et de bonneterie. Les draps viennent du Languedoc, tandis que les étoffes de soie, d'or et d'argent, les rubans, les bas, les chapeaux sont fabriqués à Lyon. La péninsule ibérique est d'ailleurs à cette époque la principale cliente des marchands-fabricants et des négociants lyonnais qui y réalisent, pour les uns, plus de la moitié de leurs ventes et, pour les autres, un quart de leurs affaires ${ }^{18}$. D'ailleurs, parmi les soixante-dix maisons françaises de com-

13. Arch. nat., Aff. étr. BI 277, lettre de Cassot du 24 mai 1763.

14. Archives Diplomatiques de Nantes, Consulat de Cadix, 2 Mi 1856, reg. 257, recueil des procès verbaux des délibérations des assemblées de la Nation, septembre 1728-juin $1778, \mathrm{f}^{\circ} 196-198$ et $\mathrm{f}^{\circ} 244-246$; 2 Mi 1856, reg. 258 , registre des procès verbaux des délibérations des assemblées de la Nation, juin 1778-août 1791, f f $^{5}$-7.

15. On peut se reporter à la généalogie des Magon de Cadix publiée par LESPAGNoL, André, Messieurs de Saint-Malo, une élite négociante au temps de Louis XIV, Rennes, PUR, 1997, p. 447.

16. CHAMBOREDON, Robert, ForniER, Simon, Arnail, Les Français en Espagne à l'époque moderne (XVI'-XVIII siècle), Paris, CNRS, 1990, p. 197-214.

17. ZYLBERBERG, Michel, Une si douce domination..., op. cit., p. 75-76.

18. GARDEN, Maurice, "Les aires du commerce lyonnais au XVIII ${ }^{\mathrm{e}}$ siècle ", Aires et structures du commerce français au XVIII siècle, Paris, CNRS, 1973, p. 265-289. 
merce et de négoce en gros recensées par le consul De Mongelas en 1777, douze ont été fondées par des Lyonnais ou en comptent un parmi leurs associés ${ }^{19}$, une d'entre elles, Jean-Baptiste Vande et Favre, appartenant même à la première classe de la Nation aux côtés des Magon, Le Couteulx et autres Fornier ${ }^{20}$.

Groupe le plus nombreux, la colonie française est aussi le groupe le mieux organisé, dominé et représenté officiellement par un Corps de Nation auquel appartiennent en 1771 les représentants des 154 principales maisons de commerce établies dans la ville ${ }^{21}$. Sous la présidence du consul, il représente et défend les intérêts du commerce français dans le port andalou et, chaque année, désigne en son sein deux députés qui en sont les porte-parole directs, autant auprès des autorités locales que des autorités françaises. Quant au consul, représentant du Roi de France dans la ville, il a auprès de la Nation des compétences en matière de police et de justice, règle les problèmes de succession, protège les intérêts de la navigation commerciale et, au quotidien, intervient dans toutes les affaires qui peuvent opposer un membre de la Nation à une autorité locale. En outre, conformément à l'ordonnance de 1681 qui définit ses fonctions, il doit adresser au ministère de la Marine une correspondance régulière pour " rendre un compte exact de toutes les affaires de [son] département ${ }^{22}$ ", ce qui en fait pour nous un observateur privilégié de la vie gaditane et de l'activité portuaire.

\section{La correspondance des consuls}

Le poste de Cadix est au XVIII ${ }^{\mathrm{e}}$ siècle l'un des plus importants de la carrière consulaire - et le mieux rémunéré ${ }^{23}$. Au cours de notre période, deux consuls se succèdent dans cette fonction : Antoine Dumazel de Puyabry et Etienne Hardy du Plessis de Mongelas. Consul depuis la mort de son père en mars 1759, De Puyabry n'est pas à Cadix quand débute notre correspondance. Il est retour en en France à la fin de l'année 1762 et ne revient qu'en mai 1764. En son absence, c'est le chancelier Louis Cassot qui tient la plume, informe le ministre de ce qui se passe dans le port andalou et doit gérer la délicate affaire des contrôles (visitas) que la douane espagnole pré-

19. Arch. nat., Aff. étr. $\mathrm{B}^{\mathrm{I}} 283 \mathrm{f}^{\circ} 5$, " liste des négociants, boutiquiers, artisans, domestiques et autres sujets du Roy établis et résidents présentement à Cadiz, sous la protection de Sa Majesté ", du 20 janvier 1777 (f $\left.{ }^{\circ} 28\right)$.

20. Archives Diplomatiques de Nantes, Consulat de Cadix, 2 Mi 1856, reg. 258, f ${ }^{\circ} 5-7$, délibérations des assemblées de la Nation, rôle de cotisation du 17 juin 1778.

21. Arch. nat., Aff. étr. B $B^{\mathrm{I}} 279$, copie d'une lettre écrite par les députés de la Nation française à Cadiz au marquis d'Ossun, ambassadeur du Roi à Madrid (16 avril 1771), jointe à une lettre du consul De Puyabry du 23 avril 1771; les députés recensent alors 72 maisons de négoce, 32 maisons de détaillants et 50 " artistes divers et marchands de mode ".

22. MÉzin, Anne, Les Consuls de France au siècle des Lumières (1715-1792), Paris, ministère des Affaires étrangères, 1997, p. 42.

23. Anne Mézin évalue le revenu annuel du consul de Cadix à 30000 ou 40000 l par an, Les Consuls de France..., op. cit. 
tend imposer à bord des navires français. Présent à Cadix depuis 1737, Cassot avait déjà secondé le consul Bigodet de Varennes et, fort de son expérience et du soutien de l'ambassadeur du Roi à Madrid, avait même été pressenti pour le Consulat en 1758, avant que ne lui soit préféré De Puyabry père, ancien consul à Carthagène puis à Barcelone. Quand il se retire en septembre 1764, prétextant des raisons de santé qui ne lui permettent plus " de vaquer avec la même activité aux affaires de la chancellerie ", la Nation décide à l'unanimité de lui octroyer " une pension viagère de 250 piastres par an ${ }^{24}$ ". En 1775, Antoine de Puyabry se retire à Périgueux, sa ville natale, et le Roi nomme Du Plessis de Mongelas pour le remplacer. Cet ancien officier originaire de Nantes et qui a servi de longues années dans le Nord (au Danemark, en Suède, en Prusse, en Russie) ainsi qu'en Hollande, a profité de ses précédentes affectations pour s'initier aux choses du commerce; aussi en fait-il le principal sujet de sa correspondance ave le ministre, multipliant états de la navigation, mémoires sur le commerce, propositions diverses pour développer les échanges avec l'Espagne et l'Amérique et renforcer sinon conforter le pois économique de la Nation française à Cadix. De Mongelas ne prenant ses fonctions qu'en janvier 1776, l'intérim est assuré pendant pratiquement toute l'année 1775 par JeanBaptiste Poirel qui est installé dans ses fonctions de consul temporaire par une Assemblée générale de la Nation réunie en mars $1775^{25}$. Vice-consul depuis 1769, il avait été auparavant secrétaire du consulat puis chancelier après le retrait de Cassot. Quand en mars 1778 De Mongelas regagne la France pour quelques mois, sa signature réapparaît au bas des lettres ${ }^{26}$.

Entre 1763 et 1778, les consuls de France à Cadix et ceux qui les secondent adressent plusieurs centaines de lettres, d'abord au ministère des Affaires étrangères puis à celui de la Marine ${ }^{27}$, au rythme moyen de deux ou trois lettres hebdomadaires. Cette abondante correspondance est conservée aux archives Nationales, dans la sous-série $\mathrm{B}^{\mathrm{I}}$ du fonds des Affaires étrangères, " correspondance consulaire, Cadix, 1666-1792, lettres reçues des agents consulaires réunies en registres ". Les lettres des consuls sont rassemblées en huit registres cotés B 277 (tome 67) à B 284 (tome 74) et sont assez souvent accompagnés de copies de courriers envoyés à l'ambassadeur du Roi à Madrid ainsi que de quelques mémoires rédigés par les députés de la Nation, lesquels portent sur l'état du commerce ou plaident pour la défense des privilèges jadis octroyés aux Français par les prédécesseurs de Charles III.

Les affaires du commerce entre la France, l'Espagne et l'Amérique espagnole sont au cœur de cette correspondance. Les consuls évoquent les

24. Arch. nat., Aff. étr., B $B^{\mathrm{I}} 277$, lettre de De Puyabry du 4 septembre 1764.

25. Arch. nat., Aff. étr. B 281 , lettre du 28 mars 1775.

26. Mézin, Anne, Les Consuls de France..., op. cit.; notices biographiques de Dumazel de Puyabry p. 257, de Du Plessis de Mongelas p. 344, de Cassot p. 179, de Poirel p. 496-497.

27. De 1761 à 1766, les consulats sont détachés du ministère de la Marine et rejoignent celui des Affaires étrangères. Mais ils retrouvent leur département d'origine quand le Duc de Praslin est nommé ministre de la Marine en avril 1766. 
mécanismes de ce commerce, les contraintes auxquelles doivent se plier ceux qui le pratiquent pour expédier leurs marchandises sur la Carrera et pour en recueillir les fruits, les activités qu'ils exercent en complément du négoce, comme le prêt à la grosse aventure ou la spéculation sur les piastres. Ils interviennent aussi longuement pour défendre les intérêts de leurs nationaux et pour réclamer le soutien de la Cour dans leurs démarches auprès des autorités espagnoles, à une époque où celles-ci entreprennent une série de réformes structurelles pour renforcer leur contrôle sur la route des Indes. Parmi les questions soulevées au cours de notre période, nous retiendrons d'abord celle des inspections conduites par la Douane à bord des navires français - mais aussi anglais et hollandais - qui entrent dans la baie de Cadix, visitas qui suscitent moult démarches des consuls entre 1763 et 1765 . Puis, à partir de 1767, les maisons françaises de la ville sont inquiétées par les prétentions du Consulat des Indes (l'Université des marchands qui commerçaient avec les Indes ou Universidad de Cargadores a Indias ${ }^{28}$ ) à vouloir contrôler leurs activités, et en particulier la façon dont certains négociants investissent leurs fonds sur la Carrera par l'intermédiaire d'hommes de paille espagnols ou par le biais de leurs femmes et de leurs fils nés en Espagne. Pendant que le Consulat des Indes tente de restreindre l'accès au commerce colonial pour les étrangers, les commerçants gaditans proposent au gouvernement de Madrid d'obliger leurs confrères étrangers à intégrer leur corporation, à renoncer à leur nationalité ainsi qu'à tous les privilèges inhérents à leur condition. Dans le même temps, le rétablissement de la paix permettant la reprise des liaisons ave l'Amérique, les premiers retours favorisent une reprise du commerce des métaux précieux et, inévitablement, de la fraude qui l'a toujours accompagné. Si d'un côté les consuls tiennent régulièrement le ministre au courant des exportations de piastres, ils doivent aussi solliciter à plusieurs reprises son intervention auprès de capitaines et de marins pris la main dans le sac, en flagrant délit de contrebande, ou dénoncés comme contrebandiers. C'est dans ce contexte que surgit d'ailleurs la question des visitas, après 1775 .

En mettant en évidence ces quelques questions du renforcement des contrôles douaniers, des atteintes aux privilèges de la Nation française et du commerce des piastres, la correspondance consulaire nous renseigne aussi régulièrement sur les mouvements de navires qui ont lieu dans la baie de Cadix, sur l'armement et le retour des Flottes des Indes, sur les arrivées

28. Antonio Garcia-Baquero présente ce Consulat comme « une corporation de tous les négociants intéressés dans le trafic de la Carrera, [...] un tribunal spécialisé chargé de résoudre, plus rapidement et à moindre qu'à la Casa de Contratacion, tous les litiges surgis entre ses membres dans l'exercice de leurs activités commerciales ". Il rappelle également que " une de ses fonctions primordiales était de regrouper et de représenter les marchands de la Carrera dans la défense de leurs droits, aussi bien face aux tentatives de pénétration d'autres commerçants - en particulier les étrangers [...] - que face à la Couronne ". GARCIA-BAQUERO GONZALEZ, Antonio, La Carrera de Indias, histoire du commerce hispano-américain (XVI'-XVII e siècles), Paris, Desjonquères, 1997, p. 59. 
de vaisseaux de registre revenant de la mer du Sud, de La Vera Cruz ou de La Havane, sur la composition des escadres espagnoles comme celle qui, en 1775, participe à la campagne contre Alger. Enfin, au détour de nombreuses lettres, le lecteur peut aussi prendre le pouls de la vie gaditane, partager avec les habitants de Cadix quelques frayeurs comme celles consécutives aux tremblements de terre du 6 avril 1772 et du 12 avril $1773^{29}$; faire face avec eux aux rigueurs de l'hiver 1765-1766, quand « le froid s'est fait sentir extraordinairement " et qu'" on a vu, ce qui n'arrive pas à Cadix, ou du moins très rarement, de la glace et de la neige ${ }^{30}$ "; affronter quelques tempêtes comme celle du 20 février 1766 dont la force est telle qu'on fit " des prières publiques dans plusieurs églises de la ville ${ }^{31}$ ", celle du 30 janvier 1772 qui provoqua l'échouage de plusieurs navires et coûta la vie à plusieurs marins ${ }^{32}$, ou celle du 5 janvier 1778, " ouragan des plus violents qui [...] rendit la mer furieuse ", causant de nouveaux naufrages et de nombreuses noyades ${ }^{33}$. On peut également partager l'émotion de la Nation française quand lui parviennent les nouvelles de la mort du Dauphin en janvier 1766, puis du décès de Louis XV en mai 1774, disparitions royales après lesquelles toute la Nation prend le deuil pour trois mois et organise de somptueuses et coûteuses pompes funèbres ${ }^{34}$. On peut enfin relever quelques faits divers et parfois en sourire, comme par exemple à la lecture de la lettre de De Mongelas qui raconte comment, en 1776, le samedi de la Semaine Sainte, un artisan espagnol offensa ouvertement toute la Nation française en suspendant devant sa porte un mannequin assimilant Judas à un Français :

" Il est d'usage parmi le peuple d'Espagne, le samedi de la Semaine Sainte, de faire un homme de paille représentant Judas. Cette figure est pendue au milieu des rues et reçoit toutes les imprécations d'une populace guidée par le fanatisme le plus outré. Un tailleur s'avisa d'attacher à la figure qui pendait devant sa porte les écriteaux les plus inconsidérés et les plus injurieux à la Nation française. D'un côté était écrit en espagnol " observez que je suis Judas et Français pour trahir mon maître "; et de l'autre "regardez bien que je suis Français ». La foule assemblée et vomissant d'injures contre la Nation française arrête tous les Français qui passaient dans cette rue et leur présente avec raillerie et avec des gestes brutaux l'objet de son mépris, de sa dérision, et la comparaison qu'elle en fait avec la Nation française. L'insulte que recevaient ces Français, leur indignation justement excitée, les engagea à recourir à moi pour arrêter un scandale qui commençait déjà à produire des animosités et des querelles prêtes à éclater et à causer dans la ville une rumeur considérable. Je fus sur le champ chez le gouverneur. Je lui deman-

29. Arch. nat., Aff. étr. B 280, lettres de De Puyabry du 10 avril 1772 et du 13 avril 1773. 30. Arch. nat., Aff. étr. B 278, lettre de De Puyabry du 25 février 1766.

31. Idem.

32. Arch. nat., Aff. étr. B 280 , lettre de De Puyabry du 31 janvier 1772.

33. Arch. nat., Aff. étr. B ${ }^{\mathrm{I}} 284$, lettre de De Mongelas du 6 janvier 1778.

34. Arch. nat., Aff. étr. B 278 , lettre de De Puyabry du 17 janvier 1766 ; $B^{\mathrm{I}} 280$, lettre de De Puyabry du 27 mai 1774; B 281, lettre de De Puyabry du 7 janvier 1775 qui précise que le coût des cérémonies organisées à l'occasion du décès du roi s'élève à « 43313 réaux de platte $12 / 16 "$. 
dai avec insistance de faire enlever cette figure et de punir sévèrement le propriétaire de la maison où elle était pendue [...]. M. le Gouverneur, outré de cet accident, ordonna sur le champ d'enlever la figure de Judas et fit conduire en prison le tailleur, me promit d'instruire son procès et de le faire chasser de la ville ${ }^{35}$."

Finalement, De Mongelas intervint en faveur du provocateur et obtint, après trois jours d'incarcération, sa remise en liberté à condition qu'il s'excusât devant la Nation et payât " une amende d'une pistole d'or applicable aux pauvres ${ }^{36}$ ".

\section{“ L'Espagne ne peut se passer de nous ${ }^{37}$ "}

Pendant toute notre période, les consuls de France à Cadix distillent dans leur correspondance des informations sur le commerce gaditan, adressent des rapports au ministre de la Marine ou lui transmettent des mémoires rédigés par les députés de la Nation. Le consul De Mongelas se montre particulièrement attentif aux affaires du commerce et, dès sa prise de fonction, fait un constat sans équivoque sur la supériorité du négoce français et sur la nécessité qu'il y a tant à le favoriser qu'à le protéger :

"L'Espagne ne peut se passer de nous [...]. Notre activité, notre génie inventif, la hardiesse de nos entreprises l'emportent toujours sur la lenteur, sur l'imagination et sur l'irrésolution de l'Espagnol [...]. L'Espagne n'est pas puissance à redouter. Elle n'a que l'ombre de sa force. La splendeur de son opulence ne doit pas nous éblouir. La faiblesse de son gouvernement, sa mauvaise administration, l'indolence naturelle à la nation lui font perdre beaucoup des avantages qu'elle pourrait retirer de ses riches possessions [...]. Il est entré en cette baie, en 1775, 178 navires français; en 1774, il n'en était entré que 164 [...]. Notre morue, nos harengs, nos étoffes de laine et autres branches de commerce sont des objets très intéressants. Ce n'est que par la protection que le commerce pourra s'accroître. Y a-t-il un endroit plus propre et plus favorable pour le nôtre que l'Espagne ${ }^{38}$ ? "

C'est que, à l'en croire, la conjoncture est alors des plus favorable à l'essor du commerce français avec l'Espagne, d'autant que l'Angleterre est aux prises avec ses colonies d'Amérique du Nord et que les navires hollandais sont devenus une des proies favorites des pirates barbaresques. Bon connaisseur du Nord, il n'hésite pas non plus à prêcher pour un recadrage du commerce maritime français sur l'Europe atlantique, du détroit de Gibraltar au détroit du Sund et au-delà, ainsi que sur le grand cabotage.

« L'Angleterre occupée dans ses colonies, le commerce de celles-ci entièrement interrompu, la Hollande en guerre avec les Barbaresques, l'Espagne qui emploie tous ses vaisseaux marchands pour son expédition et pour son commerce de l'Amérique, tout semble nous faire espérer de profiter du

35. Arch. nat., Aff. étr. B 282 f $^{\circ}$ 29, lettre de De Mongelas du 9 avril 1776.

36. Arch. nat., Aff. étr. $\mathrm{B}^{\mathrm{I}} 282 \mathrm{f}^{\circ}$ 29, lettre de De Mongelas du 9 avril 1776.

37. Arch. nat., Aff. étr. $\mathrm{B}^{\mathrm{l}} 282 \mathrm{f}^{\circ}$ 5, lettre de De Mongelas du 10 janvier 1776.

38. Idem. 
calme et de la tranquillité dont notre gouvernement jouit. L'avantage que nous pouvons retirer est si grand qu'il serait peut-être un mal de ne pas le saisir, et peut-être aussi l'instant favorable est venu de donner à notre navigation de nouveaux débouchés et l'élever sur la décadence de celle de nos rivaux. Longtemps la Hollande a fait le cabotage de l'Europe. C'est en approvisionnant ses marchés des productions de toutes les nations qu'elle doit sa richesse et l'avantage de voir aujourd'hui qu'on ne peut pas se passer d'elle dans le commerce en général. Mais la France, qui la première avait ouvert à cette nation la facilité de porter ses marchandises dans le Nord, a su insensiblement se conserver un bien réel. Une navigation active, un fret modique ont eu la préférence, et son cabotage est devenu et devient tous les jours une école de marine. La lenteur des Hollandais, la pesanteur de leurs navires les éloignent visiblement de cette supériorité qu'ils ont eu, et plus que tout cela le haut prix des assurances sur leurs cargaisons. Aujourd'hui on ne trouve point à assurer à $6 \%$ sur un vaisseau hollandais venant de Hambourg à Cadix. Nos navires commencent à se montrer dans cette partie du Nord et à y être préférés même aux Danois. Il ne m'est permis, Monseigneur, que de vous représenter les avantages qu'ils retirent d'exporter dans le Nord tous les fruits et denrées d'Espagne, et je n'ose approfondir les branches de commerce qu'offre la Baltique [...]. La France réunit toutes les facilités pour le cabotage et cultive avec succès une branche de commerce qui établit l'abondance chez toutes les nations de ce qui leur manque et les débarrasse du superflu de leurs richesses. Elle occupe une grande quantité de vaisseaux. C'est elle qui entretiendra sans cesse dans les différentes places de l'Europe les objets de nos fabriques en les renouvelant sans cesse. Le cabotage de l'Espagne, l'exportation de ses produits sont considérables. Nous y participons déjà. Mais l'éloignement que nous voyons aujourd'hui des vaisseaux des autres nations doit nous l'assurer ${ }^{39}$."

Le commerce d'Espagne a connu un réel essor au cours de la décennie écoulée puisque, le 4 février 1766, le consul De Puyabry répondait au ministre qui lui avait demandé un " état général de tous les navires qui sont entrés et sortis dans le courant de l'année [1765] " que 100 navires marchands français étaient passés par Cadix et que 73 d'entre eux y avaient fait du commerce ${ }^{40}$, contre 84 en $1763^{41}$. En moins de quinze ans le trafic a donc plus que triplé en nombre de navires, d'où l'enthousiasme et les ambitions de De Mongelas.

La correspondance consulaire nous permet d'apercevoir ce cabotage atlantique et méditerranéen, notamment dans les "Nouvelles de la mer " transmises régulièrement au ministre de la Marine. Toutefois, ces " nouvelles " nous informent davantage sur les naufrages et autres fortunes de mer que sur les bateaux arrivés sans dommages ${ }^{42}$. Mais on peut constater que, parmi les ports français qui entretiennent des relations avec Cadix,

39. Arch. nat., Aff. étr. $B^{\mathrm{I}} 282 \mathrm{f}^{\circ}$ 82, lettre de De Mongelas du 24 septembre 1776.

40. Arch. nat., Aff. étr. B 278 , lettre de De Puyabry du 4 février 1766.

41. Arch. nat., Aff. étr. B $\mathrm{B}^{\mathrm{I}} 277$, lettre de Cassot du 20 janvier 1764.

42. Pour une approche du cabotage entre la France et Cadix, signalons le fonds certainement intéressant constitué par les " consulats " et déclarations des capitaines faites devant le consul de Cadix, conservés dans les actes de chancellerie du Consulat aux Archives diplomatiques de Nantes. 
les noms de Marseille, Le Havre, Nantes et Saint-Malo reviennent le plus souvent. C'est vers ce dernier port breton que se dirigeait Le Saint-Vincent quand il fit naufrage le 30 janvier 1772, entraînant la perte d'une cargaison de " provisions et comestibles ${ }^{43}$ ". La même tempête qui souffla sur la baie cette nuit de janvier provoqua aussi l'échouage du Nouveau Mercure du Havre, chargé de vin et d'eau de vie, et de L'Aimable Catherine, un navire nantais chargé de " 400 barils de farine, de quelques autres provisions et de vingt ballots ou environ de toileries " et dont seulement deux matelots survécurent au chavirage ${ }^{44}$. Quant au brigantin marseillais L'Heureux Clairon qui regagnait son port d'attache " avec une riche cargaison ", il fut abandonné par son équipage après qu'un incendie se soit déclenché dans son poste de cuisine dans la soirée du 6 septembre $1773^{45}$. Le consul informe également le ministre sur les mouvements de navires espagnols et d'autres pavillons. Ainsi De Puyabry signale-t-il le naufrage de deux bâtiments anglais lors de la violente tempête du 20 février $1766^{46}$, ou le passage de trois navires suédois en février $1769^{47}$. De son côté, Poirel décrit minutieusement l'escadre de 118 voiles que les Espagnols arment dans la baie au début du mois de mai 1775 après avoir déclaré la guerre à Alger ${ }^{48}$. Mais ce sont surtout les mouvements des vaisseaux de la Flotte, des avisos et des navires de registre (registros sueltos) qui appareillent vers l'Amérique ou qui en reviennent qui sont systématiquement rapportés avec, quand cela est possible, une description des cargaisons de retour.

\section{Le commerce des Français en Amérique}

C'est que Cadix est d'abord, et ce depuis plus d'un siècle, l'antichambre de l'immense empire colonial espagnol. Entre 1763 et 1778 le trafic du port andalou est à son apogée. Antonio Garcia-Baquero a recensé 695 appareillages pour les Indes entre ces deux dates, ainsi que 766 retours, $70 \%$ des vaisseaux au départ et $54 \%$ à l'arrivée étant des navires marchands ${ }^{49}$. Aussi n'est-il pas étonnant que le commerce avec l'Amérique espagnole, dans lequel les Français sont indirectement très impliqués, ait fait l'objet, avant même le commerce franco-espagnol, d'une abondante correspondance. Au cours de notre période, quatre Flottes prennent la mer pour La Vera Cruz en février 1765, décembre 1768, mai 1772 et mai 1776. Les consuls en informent leur ministre et De Mongelas, qui présente le chargement de la dernière Flotte comme « le plus considérable et le plus riche qui ait jamais

43. Arch. nat., Aff. étr. B 280, lettre de De Puyabru du 7 février 1772.

44. Arch. nat., Aff. étr. B ${ }^{\mathrm{I}}$ 280, lettre de De Puyabry du 31 janvier 1772.

45. Arch. nat., Aff. étr. B 280 , lettre de De Puyabry du 15 octobre 1773.

46. Arch. nat., Aff. étr. B 278 , lettre de De Puyabry du 25 février 1766.

47. Arch. nat., Aff. étr. B ${ }^{\mathrm{I}} 279$, lettre de De Puyabry du 21 février 1769.

48. Arch. nat., Aff. étr. B 281 , lettres de Poirel des 5 et 9 mai 1775.

49. Nous obtenons ces chiffres et avançons ces estimations à partir des tableaux dressés parx GarCia-BAQUero GonZalez, Antonio, Cadiz y el Atlantico (1717-1778) el comercio colonial espanol bajo el monopolio gaditano, Séville, escuela de estudios hispano-americanos de Sevilla, t. II, p. 79 et 125. 
été exporté d'ici pour la Nouvelle-Espagne ", relève sur un total de dix-sept bateaux la présence de " cinq gros navires marchands [...] tous chargés de diverses marchandises sèches et liquides, escortés par deux vaisseaux de guerre de 60 canons chacun ${ }^{50}$ ". Les retours des Indes sont eux aussi régulièrement signalés et les cargaisons parfois décrites, comme celle du SaintPierre qui, en décembre 1763, rapporte du sucre et du tabac de SaintDomingue et de Cuba, ou celle de La Constance, un navire de registre qui revient de Carthagène et de La Havane en décembre 1769 :

"Sa cargaison consiste en 850000 piastres fortes en or et argent monnayé et façonné, 414 surons de cacao de La Magdeleine, 375 caisses de sucre, 27 arrobes d'écaille, 4668 arrobes de quinquina, 1000 cuirs en poil et en 200 quintaux de bois de Campêche ${ }^{51}$."

Parfois, le consul joint à son courrier une note imprimée par la Casa de Contratacion et qui donne le détail des cargaisons rapportées à l'image de celle que De Puyabry joint à sa lettre du 22 mai 1772, le surlendemain du retour de La Vera Cruz et de La Havane de trois vaisseaux, le Raphaël, le Saint-Pierre et la Conception ${ }^{52}$. Sur la période, quatre Flottes reviennent de La Vera Cruz, les trois premières - de quatre navires chacune - en mars 1763, juillet 1770 et mars 1774 , et la dernière - de neuf navires - en juin $1778^{53}$.

Le commerce français avec l'Amérique espagnole porte principalement sur les toiles et les étoffes. Beaucoup de marchands français de Cadix sont les consignataires de grandes maisons de la France toilière de l'Ouest ou des fabriques lyonnaises ou languedociennes. Nous connaissons particulièrement bien parmi eux Antoine Granjean, correspondant de la maison Linossier de Lyon ${ }^{54}$. L'activité de ces marchands est rythmée par les départs et les retours des Flottes porteuses d'informations sur l'état des marchés américains. Ainsi, en juin 1767, De Puyabry fait part de nouvelles peu encourageantes pour le commerce du Pérou rapportées par un navire arrivé de Carthagène ${ }^{55}$. Par contre, dix ans plus tard, on apprend de De Mongelas que les marchandises apportées à La Vera Cruz par la Flotte de 1776 s'y sont bien vendues et que les toiles de Bretagne ont eu un grand succès puisqu'elles " ont été de préférence enlevées à celles d'Allemagne dont le prix était extrêmement bas et dont la qualité commen[çait] enfin à désabuser l'acheteur ${ }^{56}$ ". Dans cette même lettre, le consul ne cache pas cependant ses inquiétudes à voir se développer le commerce interlope entre la Nouvelle-Espagne et les colonies anglaises, alors que sept navires

50. Arch. nat., Aff. étr. $\mathrm{B}^{\mathrm{I}}$ 282, lettre de De Mongelas du 10 mai 1776.

51. Arch. nat., Aff. étr. B 279 , lettre de De Puyabry du 29 décembre 1769.

52. Arch. nat., Aff. étr. B 280 , lettre de De Puyabry du 12 mai 1772.

53. Antonio Garcia-Baquero Gonzalez, Cadiz y el Atlantico..., op. cit., t. I, p. 280-281, t. II, p. 94.

54. DoRNIC, François, «Le commerce des Français à Cadix d'après les papiers d'Antoine Granjean (1752-1774) ", Annales ESC, Paris, avril-juin 1954, p. 311 à 327; VIAL, Pierre, Un négociant français à Cadix : Antoine Granjean (1752-1775), Université de Lyon, 1964, $191 \mathrm{p}$.

55. Arch. nat., Aff. étr. $B^{\mathrm{I}}$ 278, lettre de De Puyabry du 23 juin 1767.

56. Arch. nat., Aff. étr. $B^{\mathrm{I}} 283$ f 13, lettre de De Mongelas du 11 février 1777. 
de La Havane ont été arraisonnés dans le port de Vera Cruz pour avoir pratiqué " une contrebande immense de marchandises anglaises ":

"On assure que depuis cinq ans il s'introduit en fraude dans ces possessions espagnoles par cette même voie la cargaison de plus de trois cent petits bâtiments [...]. L'Espagne n'a pas de plus grand ennemi que le commerce interlope. Il porte un coup sensible à toutes les fabriques d'Europe ${ }^{57}$. "

En septembre 1770, De Puyabry explique les mécanismes du commerce français en Amérique espagnole :

"En même temps que nous n'avons aucun droit positif à ce commerce, la Cour de Madrid n'ignore pas que de temps immémorial nous en avons eu un, secret à la vérité, mais fondé sur le principe le moins équivoque, celui de la propre impuissance de ses sujets pour le faire par eux seuls. À ce premier droit nous en joignons un autre, sacré de lui-même, inaltérable et fondé sur celui même des gens, c'est celui du commerce relatif que nous faisons ici même avec ses mêmes sujets [...]. Le commerce local de Cadix consiste en achats et en ventes des Espagnols à nous. La plupart des achats qu'ils font de nous [...] sont à longs crédits de 12 à 30 et même 36 mois, en sorte qu'à la faveur de ces longs termes, les Espagnols ont le loisir de passer à leurs Indes, d'y vendre leurs effets et d'en employer ensuite le produit à nous payer, c'est-à-dire lorsque le succès de leur voyage leur permet de la faire ainsi, ce qui n'arrive pas à beaucoup près toujours. Ces achats sont souvent stipulés payables à leur retour, soit à prime de terre ou de mer, genre de négociations que les lois permettent aux étrangers. Mais si les affaires retiennent ces acheteurs aux Indes, ils nous remettent alors nos fonds aux consignations que nous leur avons indiquées, quelque fondés que nous serions dans ces sortes de cas de les faire revenir à la nôtre puisque, dans la plus exacte vérité, ce n'est là commercer qu'à Cadix et non aux Indes. Cependant, notre respect constant pour les lois du pays nous a toujours déterminé à faire adresser nos fonds à des noms espagnols. Si nous y avons employé ceux des fils et femmes d'étrangers, ce n'est qu'après avoir été longtemps témoins qu'on ne s'y opposait pas ${ }^{58}$."

Comme l'explique le consul, les Français se sont immiscés dans la Carrera de Indias par l'intermédiaire de prête-noms espagnols ou dans l'ombre de leurs épouses espagnoles ou de leurs enfants sujets du roi d'Espagne, les janizaros. Leur activité est double, voire triple. Elle consiste d'abord à vendre leurs marchandises à des chargeurs (cargadores) et ce avec un crédit qui peut aller jusqu'à trente-six mois. Ils se font alors payer en " fruits des Indes " ou en monnaie américaine ${ }^{59}$, à Cadix même ou chez un consignataire installé à La Vera Cruz ou à La Havane. Au retour des Flottes, sous couvert d'un nom espagnol, ils récupèrent les fonds, la cochenille ou le tabac qui leur reviennent et se chargent de leur revente en s'appuyant sur le réseau de leurs partenaires français, souvent installées dans la ville et dans la région d'où ils sont originaires. Enfin, avec « le genre de

57. Idem.

58. Arch. nat., Aff. étr. $B^{\mathrm{I}} 279$, lettre de De Puyabry du 14 septembre 1770.

59. Les pesos qui étaient en usage à La Vera Cruz étaient des pesos fuertes de dix réaux et non des piastres " légères" de 8 réaux comme à Cadix. 
négociation que les lois permettent aux étrangers " et qu'évoque De Puyabry dans son courrier, on touche à une autre activité qui rapporte quantité de fonds aux maisons françaises installées à Cadix : le prêt à risque ou prêt à la grosse aventure, dont le même consul décrit les mécanismes en mai 1772 :

"Le commerce étranger ayant connu par une fatale expérience les risques qu'il y avait à vendre ses marchandises à un Espagnol pour être payé ici à son retour de l'Amérique, prit le parti de les vendre ici pour en recevoir le montant à l'Amérique même par le moyen d'un Espagnol résidant dans le pays auquel il s'adressait. C'est ce qu'on appelle contrat à la grosse à risque. Ce dernier, qui recevait une commission de l'étranger, veillait sur la conduite de l'acheteur et, en conséquence de la copie ou contrat qu'on lui remettait, il retirait le produit des susdits effets, soit en argent ou en denrées. Il en était de même de l'argent à la grosse que l'on prêtait ici, ou de celui que l'on donnait pour d'autres objets relatifs au commerce ou à la navigation. Le correspondant de l'Amérique renvoyait ensuite à Cadix les fonds ou denrées, sous des noms espagnols, aux étrangers propriétaires. L'étranger même d'ici avait porté la confiance si loin, à cause de la tolérance de l'Espagne sur ce commerce, qu'il confiait ses marchandises à un Espagnol qui passait en Amérique pour les y vendre pour son compte, moyennant la commission dont on était convenu. Quelquefois cet espagnol de l'Amérique rapportait lui-même le produit des ventes; quelquefois, il avait l'ordre de l'étranger de le remettre à un Espagnol de l'Amérique qui renvoyait ensuite à Cadix les sommes à l'adresse d'un Espagnol ${ }^{60}$. "

\section{L'affaire de la Real Cedula de 1769}

Lorsque De Puyabry écrit ces lignes, les pressions se font de plus en plus pesantes sur le commerce français avec l'Amérique, avec une menace d'embargo sur les fonds en provenance des "Indes " et la volonté affichée des autorités espagnoles de contrôler davantage les activités des marchands étrangers, et des Français en particulier. Une cédule de 1769 avait en effet obligé tous ces négociants à présenter leurs contrats de société au " Consulat d'Espagne " - le consulat des Indes - et à lui faire connaître les fonds qu'ils engageaient dans la "Carrière des Indes ", une " espèce d'Inquisition " contre laquelle le consul de France et, derrière lui, toute la Nation s'étaient farouchement élevés. Il leur paraissait alors totalement incongru - non sans hypocrisie - « que le Consulat espagnol, qui n'est établi ici que pour prendre connaissance des affaires qui concernent les Indes, veuille attirer à son tribunal celles des négociants qui ne peuvent y avoir aucune relation, puisque tout le commerce aux Indes leur est interdit ${ }^{61}$ ". Il s'en était suivi en août 1770, au retour de la Flotte de La Vera Cruz, un embargo sur les fonds que la Casa de Contratacion avait refusé de remettre aux femmes espagnoles mariées avec des étrangers, aux marchands non immatriculés, aux prête-noms et à tous ceux qui servaient de paravents au négoce français. Il avait fallu une

60. Arch. nat., Aff. étr. B 280, lettre de De Puyabry de mai 1772.

61. Arch. nat., Aff. étr. B 279 , lettre de De Puyabry du 210 octobre 1769. 
intervention du marquis d'Ossun pour que la Cour de Madrid accepte enfin de " remettre ces fonds sur la Flotte "; mais elle maintint encore pendant près de trois semaines l'embargo sur " les fonds arrivés dernièrement par trois vaisseaux de Buenos Aires et de la mer du Sud ${ }^{62}$ ».

Au tout début de l'année 1772, l'ambassadeur d'Ossun informe le consul que le Roi d'Espagne n'a plus l'intention d'accepter que les étrangers continuent leurs affaires avec l'Amérique " sous des noms espagnols " et que le président de la Casa de Contratacion a reçu l'ordre de "sévir avec toute rigueur contre les Espagnols qui prêteraient leur nom pour cet embarquement ou qui leur en faciliteraient les moyens par quelqu'autre voie ${ }^{63}$ ". L'émotion est alors immense au sein de la Nation française. Ses députés, le Lyonnais Guillaume Rey et Pierre Desportes, rédigent alors un long mémoire que le consul transmet au ministre dans son courrier du 7 janvier 1772. Ils y font part de leur " consternation " non seulement de voir appliquée la cédule du 23 octobre 1769, mais aussi d'avoir reçu du marquis d'Ossun l'ordre de cesser désormais tout commerce avec les Indes, ce qui, affirment-ils, met en péril la santé de maisons dont le volume des affaires est estimé à 80 millions de livres, et fait la part belle aux Flamands, aux Allemands et aux Autrichiens, leurs principaux concurrents sur le marché des toiles. Aussi appellent-ils à la défense de leur droit à poursuivre leurs affaires de négoce et de prêt à la grosse sans être obligés " de renoncer à leur pavillon ni se faire naturaliser Espagnols " :

\begin{abstract}
"À l'arrivée de la dernière Flotte, le président de la Contratacion de Cadix ordonna [...] la détention des fonds venus à la consignation d'étrangers, fils d'étrangers, femmes espagnoles et d'autres consignations dont la propriété pourrait être soupçonnée d'appartenir à d'autres qu'à des Espagnols [...]. La situation de la Nation est des plus critique. La majeure partie de nos maisons ont depuis longtemps formé des engagements avec des négociants espagnols à qui ils ont confié des marchandises pour en être vendues pour leur compte, d'autres qui leur en ont vendues pour payer la valeur aux Indes ou au retour de la Flotte, et finalement d'autres qui leur en ont prêté différentes parties d'argent sous un intérêt convenu entre les contractants pour en être payé en Amérique ou ici suivant la nature des accords, ce que nous appelons donner de l'argent à la grosse. Notre Nation se trouve donc avoir des gros intérêts, de même que ses correspondants dans toute l'Europe ${ }^{64}$."
\end{abstract}

Le 2 mars 1772, la situation s'est décantée. Le Roi d'Espagne a accepté de modifier la cédule de 1769 mais il oblige les étrangers qui veulent continuer à commercer avec l'Amérique à ne le faire que sous le nom de cargadores espagnols " matriculés dans la Carrière des Indes ", c'est-à-dire de membres du Consulat des Indes. Cette décision, qualifiée de " bonne affaire " par De Puyabry ${ }^{65}$, permet aux Français de prendre part à la pré-

62. Arch. nat., Aff. étr. B ${ }^{\mathrm{I}}$ 279, lettre de De Puyabry du 28 août 1770.

63. Arch. nat., Aff. étr. BI 280, lettre de De Puyabry du 7 janvier 1772.

64. Arch. nat., Aff. étr. B 280 , mémoire des Députés de la Nation française joint à la lettre de De Puyabry du 7 janvier 1772.

65. Arch. nat., Aff. étr. BI 280, lettre de De Puyabry du 13 mars 1772. 
paration de la nouvelle Flotte, qui appareille en mai 1772. " Nos maisons ont contracté pour plusieurs millions d'affaires à l'occasion de la Flotte et des autres navires en charge pour les différentes parties de l'Amérique " peuvent alors se réjouir les députés Rey et Desportes qui restent toutefois inquiets face à la politique du gouvernement espagnol et de "l'intérêt qu'il prend à détruire le commerce étranger de dette place, qui porterait un coup mortel aux fabriques de France ${ }^{66}$ ". Mais, le temps de la réflexion passé, le texte remanié de la cédule ne satisfait plus tant que ça le consul dans la mesure où, écrit-il en mai, « le sort des étrangers qui concourront à l'avenir à ce commerce est moins assuré que ci devant, parce qu'elle confirme en même temps sans restriction les anciennes lois des Indes qui confisquent tous les effets appartenant à des étrangers qu'on fera passer ou revenir des Indes, quoique sous des noms espagnols ${ }^{67}$ ". Voilà qui fait peser de lourdes menaces sur la pratique si répandue et si lucrative du prêt à la grosse dont on sait qu'il peut rapporter un bénéfice s'élevant jusqu'à $50 \% 68$ :

"Cet état actuel, si opposé à celui de la tolérance dont nous jouissions avant cette époque et que le ministère de Madrid avait comme autorisé par des ordres qu'il avait donnés en différentes occasions, ne nous laisse aujourd'hui d'autre ressource pour faire ce commerce qu'en vendant nos effets à des Espagnols à longs termes pour en être payés ici à leur retour de l'Amérique, ce qui nous est permis suivant ces mêmes lois, et ce qui entraîne après soi une infinité de risques que les étrangers seront obligés de courir s'ils continuent le commerce sur ce pied là, à moins qu'ils ne veuillent s'exposer à la rigueur des anciennes lois qu'on vient de renouveler, en le faisant comme du temps de la tolérance et avant que ces deux cédules eussent eu lieu, ce qui ne serait pas prudent ${ }^{69}$."

Dans les jours qui suivent, enfin soulagés d'avoir vu partir la Flotte, les députés reprennent à nouveau la plume pour évoquer les conséquences que la " funeste cédule " pourrait avoir sur leurs affaires et sur l'économie du royaume de France, et pour obtenir du ministre un soutien plus ferme et une démarche en leur faveur auprès de la Cour de Madrid :

"Nous serions contraints de renoncer à toute espèce d'affaire pour les Indes, ce qui ferait perdre à l'État les grands et puissants avantages qu'il retire de cette branche de commerce lucrative, et ce qui occasionnerait une diminution aussi nuisible que considérable dans la masse totale de ses espèces. Il en résulterait d'ailleurs un tort irréparable pour ses fabriques et ses manufactures, dont le dépassement irait porter la langueur à tous les rameaux qui en dépendent ${ }^{70}$."

66. Arch. nat., Aff. étr. B 280 , mémoire des députés de la Nation d'avril 1772.

67. Arch. nat., Aff. étr. Bl 280, lettre de De Puyabry du 2 (?) mai 1772.

68. GARCIA-BAQUero GonZAlEZ, Antonio, La Carrera de Indias..., op. cit.

69. Arch. nat., Aff. étr. Bl 280, lettre de De Puyabry du 2 (?) mai 1772.

70. Arch. nat., Aff. étr. B $\mathrm{B}^{\mathrm{I}}$ 280, mémoire des députés de la Nation du 2 juin 1772. 


\section{Les Français et le commerce libre}

Par un décret du 16 octobre 1765, Charles III accorde à ses sujets le droit de commercer librement " avec les isles de Saint-Domingue, de Cuba, de Porto Rico, la Marguerite et la Trinité ${ }^{71}$ ». Cette première entorse au monopole ne semble pas susciter d'engouement particulier chez les négociants français, bien au contraire. Il semble en effet que cette nouvelle liberté de commerce avec les Antilles espagnoles soit " loin d'être favorable au commerce " puisque les marchandises "sèches et volumineuses " - qui constituent l'essentiel des chargements effectués par les Français - " paieront à l'avenir environ $4 \%$ de plus qu'avent et les riches comme dorures et soieries sont encore plus chargées ${ }^{72}$ ". On croit alors plutôt au sein de la Nation française que le commerce libre profitera surtout aux contrebandiers des Antilles françaises, anglaises et hollandaises :

"On ne pense pas que la nouvelle ordonnance puisse faire aucun tort à nos manufactures. On a dans l'idée qu'il se consommera toujours et à peu près la même quantité de marchandises dans les isles. On craint que l'augmentation des droits sur celles qui seront embarquées dans les ports d'Espagne n'oblige les habitants de ces colonies de s'en procurer par une voie qui est assez familière dans ces ports ${ }^{73}$."

En février 1778, De Mongelas informe le ministre que la liberté de commerce est étendue au port de Buenos Aires et que " les ports d'Espagne désignés par Sa Majesté Catholique pour participer à cette liberté de commerce sont Cadix, Séville, Santander, La Corogne, Gijon, Malaga, Carthagène, Alicante et Barcelone ${ }^{74}$ ". La perspective de ces nouvelles concurrences n'émeut guère, et ce, bien qu'on annonce simultanément " qu'il y a un autre [édit] actuellement sous presse portant la même concession pour Lima ${ }^{75}$ ". Le vice-consul Poirel écrit d'ailleurs que cela « n'apportera aucun changement notable ni préjudiciable au commerce de Cadix, soit pour les étrangers soit pour les naturels du pays ", du moins pas avant quelque temps :

" [...] S'il en résulte du changement, ce ne sera tout au plus que dans quelques années, non seulement à cause du défaut de capitalistes qui fussent en état d'entreprendre es expéditions dans les ports d'Espagne désignés pour participer à la liberté de ce commerce, mais aussi parce que ces mêmes capitalistes se trouvent réunis à Cadix qui, depuis longtemps, ou pour mieux dire de tout temps, a été le centre de toutes les expéditions pour l'Amérique espagnole. Et que d'ailleurs la plupart des fruits qu'on a coutume d'exporter d'ici pour Buenos Aires provenant des productions de l'Andalousie, tels que les huiles, les vins et eau de vie, qui font un des objets les plus considérables des cargaisons destinées pour cette partie de l'Amérique, il y a lieu de croire que ce sera toujours à Cadix que se fera le plus grand nombre d'expéditions, et les plus considérables ${ }^{76}$."

71. Arch. nat., Aff. étr. B 278, lettre de De Puyabry du 12 novembre 1765.

72. Arch. nat., Aff. étr. B 278, lettre de De Puyabry du 7 mars 1766.

73. Idem.

74. Arch. nat., Aff. étr. B 284, lettre de De Mongelas du 17 février 1778.

75. Idem.

76. Arch. nat., Aff. étr. BI 284, lettre de Poirel du 3 avril 1778. 
Bon connaisseur de l'économie gaditane et du commerce avec l'Amérique espagnole, Poirel ne se trompe pas. En effet, même si la politique de liberté commerciale cherchait bien à briser le système contraignant du port unique au profit d'une quinzaine d'autres ports de la péninsule et des Canaries ${ }^{77}$, les habitudes, les positions acquises et l'expérience des cargadores gaditans permettent au port andalou de conserver le leadership dans le commerce avec les Indes espagnoles et d'assurer encore, entre 1778 et 1796, $77 \%$ du total des exportations espagnoles vers l'Amérique et $84 \%$ des importations ${ }^{78}$.

\section{Le trafic des piastres : de la nécessité de frauder... avec discrétion}

Lorsque la paix est rétablie en 1763 , les négociants français se plaignent, "faute de permissions ", de n'avoir que " peu de fonds à envoyer en France ". Cela fait pourtant près d'un an que le marquis d'Ossun a obtenu de la Cour de Madrid " une permission pour que notre commerce d'ici puisse extraire par terre et envoyer en France un million de piastres fortes, avec la circonstance qu'on devra tenir le secret, pour lui éviter plaintes que les autres Nations ne manqueraient pas de lui faire ${ }^{79} "$. Ainsi, avec l'appui de l'ambassadeur et du consul, les maisons Chancel, Pache \& Silvestre, et Cayla Solier Cabannes \& Jugla obtiennent-elles du marquis d'Esquilache les autorisations nécessaires pour expédier 210000 piastres, ainsi qu'un engagement de la part du ministre comme quoi toute demande qui lui serait adressée " en droiture ou par l'entremise de quelqu'un de ses correspondants serait accordée sans difficultés ${ }^{80}$ ". Mais, en raison de " l'abondance des piastres en France [qui rendait] ces matières bon marché ${ }^{81}$ ", les bénéfices restent limités et il faut donc attendre encore quelques mois avant de voir véritablement [re]démarrer le trafic des métaux précieux, et principalement celui de l'argent, que ce soit sous la forme de lingots ou de piastres. Ce sont d'abord quelques vaisseaux de la Compagnie des Indes Orientales qui font escale dans la baie de Cadix. Le Penthièvre et L'Éléphant appareillent pour la Chine le 23 mars 1764 après avoir embarqué environ 300000 piastres $^{82}$; puis, en février 1766 , le Condé, le Duras et le Castries en chargent à leur tour " pour leurs destinations respectives de l'Inde ${ }^{83}$ ".

En juin 1767, le consul reçoit une note du ministre l'informant de l'envoi à Cadix de plusieurs navires du roi pour y charger des matières d'or et d'argent. Aussitôt, De Puyabry convoque « quelques notables de la Nation " et

77. La liste de ces ports est donnée dans Antonio Garcia-Baquero Gonzalez, La Carrera de Indias..., op. cit., p. 40.

78. Garcia-Baquero Gonzalez, Antonio, La Carrera de Indias..., op. cit., p. 240.

79. Arch. nat., Aff. étr. BI 276, lettre de De Puyabry du 13 avril 1762.

80. Arch. nat., Aff. étr. $B^{\mathrm{I}} 277$, copie d'une lettre de Cassot au marquis d'Ossun du 29 novembre 1763.

81. Arch. nat., Aff. étr. B 277 , lettre de Cassot du 29 novembre 1763.

82. Arch. nat., Aff. étr. B 277 , lettre de Cassot du 27 mars 1764.

83. Arch. nat., Aff. étr. B 278 , lettre de De Puyabry du 25 février 1766. 
ses deux députés, Kerloguen et Quentin, mais se garde de rendre la nouvelle publique " de crainte de causer des soupçons parmi les employés des Douanes et de la baie ". C'est que, explique-t-il au ministre, ce commerce " engage ceux qui le font, pour ne pas y perdre, à user de certains moyens dont on ne doit pas parler, ni donner occasion qu'on en parle ${ }^{84}$ ". Ces " moyens " sont ceux de la fraude, permanente à Cadix en raison du nombre et de la lourdeur des charges fiscales qui pèsent sur le trafic colonial et qui incitent constamment les négociants de la Carrera à prendre des chemins de traverse. La fraude prend des formes très diverses et tous y participent, capitaines et équipages, passagers des navires revenant des Indes, personnels des Douanes et de la Casa de Contratacion, gardes des portes de la mer, négociants étrangers et commerçants gaditans, etc. Il existe même un réseau de contrebandiers spécialisés dans le transport et le déchargement discret des "matières ", les metedores ${ }^{85}$. Dans la correspondance de De Puyabry, Cadix prend parfois l'allure d'une cour des miracles et toute la population semble participer de près ou de (pas très) loin à la contrebande :

" [...] Les officiers de la marine d'Espagne, ceux de la terre, les moines, les prêtres, des soldats domestiques, matelots et jusqu'aux dames, tous travaillent moyennant un tant pour cent pour porter des matières d'or et d'argent ou en monnaie bord des bâtiments de guerre des nations étrangères qui les attendent dans la baie ${ }^{86}$."

Mais les officiers français, les aumôniers, les chirurgiens et les écrivains de bord se prêtent aussi, non sans " avidité " et " avec un excès à cet exercice en recevant des négociants même les matières d'or et d'argent, faisant pendant le jour plusieurs voyages ", et ce gratuitement à la différence de leurs homologues anglais, suédois, danois et hollandais qui, eux, le font contre " une rétribution ou un présent ». Certains capitaines envoient même leurs chaloupes chercher des piastres directement à bord des navires de retour des Indes et mouillés dans la baie, ou dans les petits ports des environs ${ }^{87}$, certainement auprès des metedores.

À en croire le consul, il n'y a officiellement à cette époque que cinq maisons françaises à Cadix qui pratiquent ce commerce des matières d'or et d'argent; " les autres, en général, font si peu de choses que cela ne vaut pas la peine d'en parler ${ }^{88}$ ". Mais la réalité est certainement toute autre car, quelques mois après le passage des premiers vaisseaux du Roi, les députés de la Nation, Quentin et d'Etchegaray, demandent l'envoi de nouveaux bâtiments et assurent pouvoir charger pour un million de livres à bord de chacun d'eux ${ }^{89}$. Entre mai 1767 et mars 1768 , les quantités d'or et d'argent chargées à bord des quatorze vaisseaux du Roi envoyés à Cadix auraient

84. Arch. nat., Aff. étr. Bl 278, lettre de De Puyabry du 23 juin 1767.

85. GarCiA-BAQUero GonZalez, Antonio, La Carrera de Indias..., op. cit., p. 102 à 110.

86. Arch. nat., Aff. étr. B 278, lettre de De Puyabry du 18 décembre 1767.

87. Idem.

88. Id.

89. Arch. nat., Aff. étr. Bl 278, lettre de De Puyabry du 15 mars 1768. 
représenté une valeur totale de 24530000 livres, ces métaux précieux ayant pris la direction de Marseille, Bayonne, Brest, mais aussi Gênes et Amsterdam. Cela aurait représenté cinq fois plus que ce qui avait déjà été transporté l'année précédente, en 1766, par six autres unités de la Royale qui en avaient chargé pour 5200000 livres à destination de Gênes, Toulon, Bayonne et Brest. La plupart de ces piastres et lingots avaient par ailleurs été embarqués en fraude puisque, de l'aveu même de De Puyabry, un seizième seulement des quantités transportées aurait acquitté l'indult de $3 \%$. Et d'estimer très précisément que cette fraude aurait coûté à la Couronne d'Espagne 389156 livres et 5 sous de droits non perçus ${ }^{90}$.

Loin de désavouer les pratiques de ses compatriotes, le ministre demande toutefois au consul de veiller à ce que tous usent " de beaucoup de précautions et de sagesse pour ne pas donner de l'ombrage aux Espagnols ». De Puyabry promet alors, "pour faire dissiper les soupçons ", de demander désormais aux officiers des vaisseaux du Roi de mettre fin aux pratiques les moins discrètes, comme celle qui consistait " à passer et repasser plusieurs fois le jour à la porte de la mer ", les poches et les doublures des uniformes pleines de piastres... mais pour quelque temps seulement :

\footnotetext{
"Ensuite, lorsque la confiance paraîtra revenue [...] on pourra pour lors, lorsque l'occasion se présentera de quelques lingots d'or et d'argent, donner main pour les faire passer à la porte, avec une certaine quantité d'officiers pour imposer ${ }^{91}$."
}

Malgré une augmentation de l'indult, qui passe de 3 à $4 \%$ en avril $1768^{92}$, le ministre annonce, en juin, vouloir envoyer au cours des prochains mois cinq nouveaux vaisseaux à Cadix puisque, écrit-il dans une lettre chiffrée, " le succès qu'a eu cette exportation l'année dernière et celle-ci est trop intéressant pour que je ne profite pas de toutes les occasions qui pourront se présenter de la favoriser ${ }^{93}$ ". Aussi le trafic continue et, en octobre 1768, De Puyabry peut annoncer le chargement de 130000 livres de " matières " à bord de la frégate L'Enjouée ${ }^{94}$.

Face à l'ampleur de la fraude et à son caractère systématique, les autorités espagnoles décident, à la fin de l'année 1769, de renforcer la surveillance autour des navires étrangers ainsi qu'aux portes de la mer. De Puyabry fait alors savoir au ministre qu'il devrait être désormais plus difficile de faire charger des piastres " par la voie détournée " à bord des navires du Roi attendus à Cadix. En effet, plusieurs chaloupes avec des gardes mouillent désormais près des vaisseaux de guerre et empêchent toute embarcation de les accoster sans avoir été préalablement "visitée". Mais, comme ils n'ont pas autorité pour fouiller les chaloupes de ces navires, la fraude continue, sous leur nez:

90. Arch. nat., Aff. étr. B $\mathrm{B}^{\mathrm{I}} 278$, lettre de De Puyabry du $1^{\text {er }}$ avril 1768.

91. Arch. nat., Aff. étr. B $\mathrm{B}^{\mathrm{I}} 278$, lettre de De Puyabry du $1^{\text {er }}$ avril 1768.

92. Arch. nat., Aff. étr. B 278, lettre de De Puyabry du 8 avril 1768.

93. Arch. nat., Aff. étr. Bl 278, lettre chiffrée du ministre à De Puyabry du 20 juin 1768.

94. Arch. nat., Aff. étr. BI 278, extrait chiffré d'une lettre de De Puyabry du 7 octobre 1768. 
"Malgré pourtant toutes ces rigueurs, on trouva l'autre jour le moyen d'introduire à bord du Duras 13000 piastres forets, qu'on remit à une des tartanes qui a fait voile pour Marseille ${ }^{95}$."

Au cours de cette décennie 1770. Marseille apparaît comme la principale destination de piastres chargées pour la France. En ayant une fois de plus recours au chiffrage, comme cela devient la règle quand il s'agit d'évoquer les transferts de métaux précieux, De Puyabry évalue une première fois, de manière assez imprécise, "à environ cinq cent et tant de mille piastres fortes " les quantités embarquées sur " différents bâtiments de la Nation qui sont partis pour Marseille " au cours des six premiers mois de $1772^{96}$. Ensuite, les estimations se précisent :

- 170000 piastres fortes pendant les trois premiers mois de $1773^{97}$;

-214000 piastres fortes en avril, mai et juin $1773^{98}$;

- au cours des trois mois suivants, plus de 300000 piastres, " tant pour Marseille que pour les ports du Ponant ${ }^{99} "$;

- 160000 piastres à bord de quatre vaisseaux partis pour Marseille entre octobre et décembre $1773^{100}$;

- 880000 piastres fortes chargées sur huit navires à destination du Havre et de Marseille au cours des derniers mois de $1774^{101}$;

- 750000 piastres fortes également emportées vers Marseille et Le Havre par dix navires au cours des mois d'avril, mai et juin $1775^{102}$.

Au total, nous le voyons, ce sont au minimum 700000 piastres qui, chaque année, gagnent Marseille depuis Cadix. En avril 1777, le consul De Mongelas évalue à huit millions de piastres les quantités d'argent qui ont quitté Cadix pour différents ports français depuis le mois d'août 1775. Il affirme aussi que pour près de $90 \%$ de ces piastres - sept millions - aucun des droits imposés par la Couronne d'Espagne n'aurait été payé (ce qui représenterait pour les Douanes une perte de 70000 piastres sur la seule base de l'indult de $4 \%$ ) et plaide lui aussi en faveur de la fraude :

" Je crois que tous les gouvernements doivent fermer les yeux et protéger tacitement une contrebande qui est avantageuse par le numéraire qu'elle fait circuler et par le bien qu'elle procure à la navigation ${ }^{103}$. "

La fraude n'a donc pas disparu et quelques capitaines français tombent encore entre les mains de l'administrateur des Douanes comme le Nantais Nieulon en 1775 ou le Marseillais André Levens en 1776. Si ce dernier reconnaît avoir bien chargé en contrebande les 23000 piastres découvertes

95. Arch. nat., Aff. étr. B 279, lettre codée de De Puyabry du 30 janvier 1770.

96. Arch. nat., Aff. étr. Bl 280, lettre de De Puyabry du 14 juillet 1772.

97. Arch. nat., Aff. étr. B 280, lettre de De Puyabry du 9 avril 1773.

98. Arch. nat., Aff. étr. B 280 , lettre de De Puyabry du 2 juillet 1773.

99. Arch. nat., Aff. étr. $\mathrm{B}^{\mathrm{I}}$ 280, lettre de De Puyabry du 8 octobre 1773.

100. Arch. nat., Aff. étr. B 280 , lettre de De Puyabry du 25 janvier 1774.

101. Arch. nat., Aff. étr. $\mathrm{B}^{\mathrm{I}}$ 281, lettre de Poirel du 6 janvier 1775.

102. Arch. nat., Aff. étr. BI 281, lettre de Poirel du 7 juillet 1775.

103. Arch. nat., Aff. étr. $\mathrm{B}^{\mathrm{I}} 283$, lettre de De Mongelas du $1^{\mathrm{er}}$ avril 1777. 
à bord du David en décembre 1776, son collègue nantais avait, quant à lui, été arrêté alors qu'il regagnait son bord avec dans ses poches vingt malheureuses piastres qui, affirmait-il, lui avaient été remises en paiement d'un fret ${ }^{104}$. Cette mésaventure lui avait valu un séjour de plusieurs mois dans les geôles gaditanes malgré les nombreuses démarches entreprises auprès du gouverneur par le consul De Mongelas. Levens s'en sort à meilleur compte peut-être parce qu'il reconnaît la tentative de fraude; son navire et sa cargaison échappent à la confiscation et il est autorisé à reprendre la mer après que les députés de la Nation, Lieutaud et Le Couteulx de la Moraye, se soient portés caution pour la valeur du bateau ${ }^{105}$. Le ministre demande alors au consul d'intervenir systématiquement auprès du gouverneur de Cadix à chaque fois qu'un capitaine français serait inquiété par les autorités espagnoles. C'est ainsi qu'en octobre 1777, le brigantin nantais L'Amitié est restitué à son armateur, et son équipage libéré, un mois seulement après la découverte à son bord de 22790 piastres fortes non déclarées ${ }^{106}$; quant à son capitaine, Jacques Fromentin, il en est quitte pour une simple admonestation puisque « le ministre et surintendant général des finances espagnol " demande au consul et à son suppléant Poirel de lui signifier ainsi qu'à son second, lorsqu'ils repasseront à Cadix, qu'il leur faudra être " plus circonspects à l'avenir dans les voyages ou relâches qu'ils pourront faire dans les ports de Sa Majesté Catholique, et de n'y plus recevoir à leur bord de matières ou espèces d'or et d'argent sans au préalable en avoir vu et vérifié les dépêches qui doivent en permettre l'extraction ${ }^{107}$ ". Cette sanction plus formelle que réellement dissuasive satisfait pleinement le Consulat et la Nation :

"Cette décision et celles rendues auparavant sur deux autres affaires de même nature concernant les navires français Le David, capitaine Levent, et L'Espérance, capitaine Prévost, doivent être désormais un préjugé favorable et victorieux que nous ne manquerons pas de faire valoir avec avantage dans les cas de ce genre auxquels nous pourrions être exposés dans la suite ${ }^{108}$."

En théorie, la fraude sur les piastres est sévèrement punie puisqu'en vertu du règlement de 1760 tout bateau à bord duquel serait " trouvé de l'or ou de l'argent monnayé ou en barres " risquerait d'être confisqué et son capitaine " châtié ", de même que le ou les marchands impliqués dans la fraude. Cette mesure était déjà dénoncée en 1763 par le chancelier Cassot comme une " loi tyrannique et injuste", dangereuse pour le commerce. Non seulement elle mettait tous les négociants à la merci " de l'imprudence d'un capitaine, d'un matelot " ou " de la malice et de la friponnerie des gardes ", mais de manière générale elle n'apportait plus " aucune sûreté " à qui que

104. Arch. nat., Aff. étr. B 282 , lettre de De Mongelas du 16 février 776.

105. Arch. nat., Aff. étr. $B^{\mathrm{I}} 282$, lettres de De Mongelas des 23 février, 13 août, 20 et 24 décembre 1776 .

106. Arch. nat., Aff. étr. $B^{I} 283$, lettres de De Mongelas du 16 septembre et du 17 octobre 1777.

107. Arch. nat., Aff. étr. $\mathrm{B}^{\mathrm{I}}$ 284, lettre de Poirel du 5 mai 1778.

108. Idem. 
ce soit, capitaines qui encouraient la prison, armateurs qui prenaient le risque de perdre leurs navires et négociants de Cadix ou de France dont les marchandises pouvaient être confisquées ${ }^{109}$. Et la menace était alors d'autant plus réelle que l'Administration de la douane, qui venait de recevoir des ordres de Madrid " pour faire visiter toutes sortes de bâtiments [...] indistinctement et sans excepter la [nation] française ${ }^{110}$ ", semblait ne plus vouloir fermer les yeux sur des pratiques trop connues et sévir contre un commerce illicite qui ne manquerait pas de se développer encore plus, maintenant que la paix était rétablie et que les prohibitions sur les marchandises anglaises et portugaises étaient levées ${ }^{111}$.

De tout temps, les prétentions de la Douane à vouloir contrôler les cargaisons des navires étrangers entrant dans la baie de Cadix et à visiter ces vaisseaux pour y chercher des marchandises de contrebande ont été très mal vécues par les marchands étrangers installés dans la ville, et pas seulement par les Français. Quand en 1763 la question des visitas de la Douane resurgit, le sujet est très sensible. Aussi occupe-t-il pendant toute notre période une place importante dans la correspondance consulaire.

\section{Les réponses à la fraude : visites et perquisitions douanières}

C'est en juillet 1763 que la Cour d'Espagne décide d'imposer ces visites. Une première tentative est dénoncée par le chancelier Cassot le 12 juillet : elle a eu lieu à bord de la Sainte-Anne, de Saint-Malo, mais son capitaine s'y est opposé. Bien que le consul s'en soit immédiatement ému auprès du gouverneur de la ville et de l'ambassadeur de France à la Cour d'Espagne, l'administrateur de la Douane a refusé d'autoriser le déchargement du navire et la délivrance des marchandises aux négociants à qui elles étaient destinées $^{112}$. Le 19 août, le capitaine d'un pinque marseillaise, La Parfaite Union, qui refuse lui aussi de se soumettre à la visite se voit également interdire de décharger ${ }^{113}$. Chacun campant sur ses positions, l'inquiétude monte au sein de la Nation française d'autant que, à en croire le consul, il y a en cette fin d'été dans la baie de Cadix, alors que des armements se préparent pour la mer du Sud, " plusieurs navires très richement chargés pour la valeur de plusieurs millions de livres " et qu'il s'attend " journellement de Saint-Malo et des autres ports de France des cargaisons fort riches ${ }^{114}$ ". Mais la Cour d'Espagne n'entend pas revenir sur sa décision. Tout au plus le marquis d'Esquilache, le puissant ministre des finances de Charles III, consent-il à

109. Arch. nat., Aff. étr. B $B^{\mathrm{I}} 277$, « Observations sur le règlement de Sa Majesté Catholique de 1760 tendantes à rendre la visite des vaisseaux français, s'ils y sont assujettis, moins onéreuse et non arbitraire ", mémoire de Cassot du 14 octobre 1763.

110. Arch. nat., Aff. étr. BI 277, lettre de Cassot du 12 juillet 1763.

111. Arch. nat., Aff. étr. $\mathrm{B}^{\mathrm{I}} 276$, lettres de De Puyabry des $1^{\mathrm{er}}$ janvier et 6 juillet $1762 ; \mathrm{B}^{\mathrm{I}}$ 277, lettre de Cassot du 19 mai 1763.

112. Arch. nat., Aff. étr. BI 277, lettres de Cassot des 12 et 29 juillet 1763.

113. Arch. nat., Aff. étr. BI 277, lettre de Cassot au marquis d'Ossun du 19 août 1763.

114. Arch. nat., Aff. étr. $B^{I} 277$, lettre de Cassot au marquis d'Ossun du 11 septembre 1763. 
accepter le remplacement des visites arbitraires par des visites qui interviendraient au huitième jour après les premières opérations de déchargement, et ce conformément au règlement de 1760. " Les choses ne changent point de nature ${ }^{115}$. " Cependant, Choiseul donne des instructions au consul pour que les équipages acceptent les visites, "sans résistance [mais] sans acquiescement " de la part des autorités françaises et de leur représentant $^{116}$. Aussi Cassot rédige-t-il à l'intention du ministre, le 14 octobre 1763, un mémoire dans lequel il rappelle que le règlement du 17 décembre 1760 n'autorise «la visite ou fondeo de la Douane [qu']après les huit jours que la décharge a été commencée ", ce qui en soit était déjà une entorse à une cédule particulière de 1703 qui exemptait les navires français de toute visite, dans le respect de l'article 15 du traité de Ryswick du 20 septembre 1697. En tout cas, les négociants français de Cadix trouvent ce terme de huit jours trop court et préféreraient qu'un délai d'au moins un mois leur soit laissé car, argumentent-ils, " il vient des marchandises pour le Port-Sainte-Marie, Xérès, Séville et autres endroits d'où il faut attendre des ordres ". Ils sont donc prêts à envisager les visites de la Douane mais demandent en retour quelques compensations comme la suppression de cette loi " tyrannique et injuste " qui prononce la confiscation de tout navire à bord duquel se trouveraient de l'or ou de l'argent non déclarés, la délivrance par les autorités espagnoles de plus de permissions pour importer des métaux précieux d'Amérique et un allègement des droits sur l'argent de 3 à $2 \%{ }^{117}$.

Sitôt les ordres de Versailles connus, les premières visites ont lieu, sans opposition mais " avec le plus grand ménagement " de la part des Espagnols, à bord du Louis-Auguste de Saint-Malo le 11 octobre, puis à bord de trois autres navires en novembre ${ }^{118}$. À la fin de l'année 1763, quinze navires français ont été visités, ce qui - et le consul le reconnaît - est peu :

« Il est aisé de voir que l'on procède à cette démarche d'une façon très modérée. En effet, la totalité des visites faites aux navires des trois nations est de quarante, pendant qu'il est arrivé dans le cours de l'année dernière, suivant les listes, 483 bâtiments marchands, savoir 84 français, 252 anglais et 147 hollandais. Dans ce nombre, ce sont les Français qui ont été le moins épargnés ${ }^{119}$."

Aussi dénonce-t-il la différence de traitement qui est faite entre les Français et leurs principaux rivaux, puisque si un bateau français sur cinq a fait l'objet d'une visite de la Douane, seuls quatorze navires anglais et onze hollandais ont aussi été contrôlés, ce qui fait respectivement une proportion de un sur dix-huit et de un sur treize. Les Anglais sembleraient même

115. Arch. nat., Aff. étr. BI 277, lettre de Cassot du 23 août 1763.

116. Arch. nat., Aff. étr. B ${ }^{\mathrm{I}} 277$, lettre de Cassot au marquis d'Ossun du 20 septembre 1763. 117. Arch. nat., Aff. étr. $B^{\mathrm{I}} 277$, « Observations sur le règlement de Sa Majesté Catholique de 1760 tendantes à rendre la visite des vaisseaux français, s'ils y sont assujettis, moins onéreuse et non arbitraire ", mémoire de Cassot du 14 octobre 1763.

118. Arch. nat., Aff. étr. $\mathrm{B}^{\mathrm{I}} 277$, lettres de Cassot des 11 octobre, 18 novembre et 9 décembre 1763.

119. Arch. nat., Aff. étr. BI 277, lettre de Cassot du 20 janvier 1764. 
avoir bénéficié d'un traitement de faveur. Dans un premier temps ils s'étaient fermement opposé à la visite - et parfois de manière spectaculaire comme le capitaine de la Suzanne qui avait chargé en fraude " une partie de cent et tant de mille piastres " et qui, après avoir refusé de laisser monter à son bord le " chef des gardes de la Douane ", avait coupé ses câbles et repris la mer avant que l'officier espagnol n'ait eu le temps d'aller " demander la troupe " au gouverneur ${ }^{120}$. Après cet épisode spectaculaire, le chef des bureaux de la Douane avait pris l'habitude de se rendre à bord des navires anglais « en s'annonçant comme ami [...] pour la forme, sans faire procéder à d'autre visite que celle de politesse qui se termine souvent par un déjeuner que les capitaines lui offrent ${ }^{121}$ ". Quant aux Hollandais, ils ne paraissent pas s'être opposés aux visites et leur attitude plus conciliante leur vaut d'ailleurs un commentaire très acerbe du chancelier Cassot :

" [Les Hollandais] laissent à la France et à l'Angleterre la discussion de cette affaire, bien persuadés que si ces deux nations réussissent à jouir des exemptions de visites, il faudra qu'ils en jouissent aussi. C'est assez le système général de ces républicains de profiter de la mésintelligence des autres nations pour augmenter leur commerce et leur navigation, objet principal des soins de leur gouvernement ${ }^{122}$."

La situation semble ensuite se normaliser pendant près de dix ans, d'autant que, les autorités françaises ayant de leur côté instauré les visites douanières à bord des bateaux espagnols, Louis XV avait donné des ordres pour que les capitaines et les consuls ne s'opposent plus aux visites dans les ports d'Espagne ${ }^{123}$. Lorsque cette question réapparaît dans la correspondance consulaire en 1777-1778, c'est d'abord pour fustiger les méthodes brutales utilisées par certains officiers espagnols comme cet Escobar, administrateur des Douanes de San Lucar de Barrameda, qui visite à trois reprises L'Espérance, un navire dieppois qui avait dû faire escale dans " la rivière de San Lucar " pour réparer quelques dommages subis au cours de la violente tempête des 14 et 15 janvier 1778. Mystérieusement informé de la présence de 300000 piastres à bord du navire français, il lui impose, après deux visites infructueuses, une troisième fouille en règle :

" [Les gardes], armés de pelles et de haches, remuent tout le sable qui sert de lest, les pierres de la cale, reconnaissent et sondent les barriques d'eau, registrent tous les coins et recoins du navire et l'entrepont, examinent le bois, les câbles, les manœuvres, la chambre, la camarotte, les caisses grandes et petites, enfin poussent l'excès de leurs recherches jusqu'à enfoncer la hache et se servir de la tarière pour sonder les doublages du bâtiment [...]. Rien ne put arrêter la rage de l'administrateur et de ses subalternes ${ }^{124}$."

120. Arch. nat., Aff. étr. B 277 , lettre de Cassot du 5 août 1763.

121. Arch. nat., Aff. étr. B 277 , lettre de Cassot du 20 janvier 1764.

122. Arch. nat., Aff. étr. B B $^{\mathrm{I}}$ 277, lettre de Cassot au marquis d'Ossun du 30 août 1763.

123. Arch. nat., Aff. étr. B 277 , lettre de De Puyabry du 12 avril 1768.

124. Arch. nat., Aff. étr. B $B^{I}$ 284, lettre de De Mongelas du 3 février 1778. 
Furieux de n'avoir pu saisir que 31 guinées, 79 piastres fortes et 14 écus de six livres, Escobar aurait alors demandé au gouverneur de San Lucar de Barrameda " d'ordonner la vente du navire au plus offrant et de prononcer la peine des galères contre son capitaine et son équipage ", requête à laquelle le gouverneur ne satisfait pas, préférant appeler son subordonné à libérer les marins, à rendre son argent au capitaine, à " être plus circonspect à l'avenir et [à] ne plus blesser désormais les privilèges que les deux monarques ont accordé à leurs sujets dans leurs ports respectifs ${ }^{125}$ ". C'est également sur la base d'une dénonciation calomnieuse qu'au même moment, à Cadix, le gouverneur Xerena ordonne, en présence du consul, la fouille du Désiré. La visite n'ayant rien donné, le gouverneur décide de faire un exemple en ordonnant l'arrestation " du délateur qui avait eu l'imprudence de déclarer à l'administrateur qu'il devait se trouver 40000 piastres à bord ${ }^{126}$ ". Cette reprise des visites à bord des navires français inquiète alors fortement la Nation car, en portant préjudice à la réputation des négociants, elles menacent leurs affaires :

"Il est bien sensible d'avoir toujours des événements fâcheux et d'essuyer des vexations qui entraîneront la ruine de notre commerce [...]. Notre navigation tombe surtout pour l'exportation des fruits de l'Espagne et des Indes. On ne charge qu'avec peine sur les vaisseaux français parce qu'on sait qu'ils sont les seuls sur qui tombent les soupçons vrais ou faux de contrebande. Le mal est très grand [...]. Tous ces objets vont immanquablement détruire notre commerce et notre navigation ${ }^{127}$."

À Cadix, la fraude ne porte pas que sur l'or et l'argent. La lourdeur de la fiscalité espagnole est telle que le commerce illicite concerne également toute la gamme des marchandises exportées vers l'Amérique ainsi que les produits coloniaux comme le tabac, qui fait aussi l'objet d'un monopole royal. Ainsi c'est parce qu'ils ont " chacun une livre de tabac râpé caché dans leurs culottes " qu'un voilier et un calfat de la flûte du Roi le Gros Ventre sont arrêtés à la Porte de la mer le 10 octobre 1769. Sur intervention du consul, le gouverneur de Cadix consent à rendre les deux hommes à leur capitaine, le vicomte de Beaumont, qui avait pourtant prévenu tout son équipage qu'il était interdit de faire entrer ou sortir de la ville quoi que ce soit " sans la permission de la Douane ${ }^{128}$ ". Le capitaine du Gros Ventre s'étant engagé à présenter ses deux matelots au tribunal sitôt son retour à Cadix, le consul et l'ambassadeur s'emploient dans les semaines qui suivent à obtenir " le pardon de la Cour ", et leurs démarches aboutissent puisqu'en janvier 1770 le marquis d'Ossun informe le consul que, "Sa Majesté voulant bien pardonner les deux marins ", ceux-ci ne seront pas poursuivis ${ }^{129}$. Cela n'avait pas été le cas de Barthélemy Sucia qui avait été arrêté en 1762 aux portes de Cadix en possession, lui aussi, de tabac râpé de contrebande. Il

125. Idem.

126. Arch. nat., Aff. étr. $\mathrm{B}^{\mathrm{I}}$ 284, lettre de De Mongelas du 17 février 1778.

127. Idem, passage chiffré.

128. Arch. nat., Aff. étr. BI 279, lettre de De Puyabry au marquis d'Ossun du 17 octobre 1769.

129. Arch. nat., Aff. étr. B 279 , lettre de De Puyabry du 12 janvier 1770. 
avait été condamné en première instance à regagner la France et interdit de séjour en Espagne. Mais la Cour de Madrid, sans doute désireuse de faire un exemple, avait estimé la sanction trop clémente et, rejugé, Sucia avait lors été condamné à " cinq ans de travaux dans les châteaux d'Afrique ${ }^{130}$ ". Trouvant le verdict excessif, le chancelier Cassot était alors intervenu auprès du marquis d'Ossun pour qu'il obtienne la commutation de la peine en " exil perpétuel ${ }^{131}$ ", arguant notamment de la santé fragile de son compatriote. S'il y eut démarche de l'ambassadeur, on peut supposer qu'elle aboutit puisqu'au cours des mois suivants on ne retrouve pas trace de cette affaire ni dans les lettres de Cassot ni dans celles de De Puyabry.

Il arrive aussi que les investigations douanières débouchent sur des visites domiciliaires. En janvier 1769, la maison des frères Labadie est fouillée par un " visiteur général de la Ronde de tabac [...] sous le prétexte qu'il y avait une contrebande de tabac râpé ". La visite ne donne rien et, parce que l'officier de la Douane a agi "sans l'aveu de ses supérieurs", le consul s'empresse d'en aviser l'ambassadeur " pour obtenir de la Cour de Madrid que le sieur Charles Ocampos et Dessemblables ne puissent à l'avenir, sous quelque prétexte que cela soit, et sans y être autorisés par leurs supérieurs, aller dans les maisons de commerce pour y faire des recherches aussi vagues et aussi peu fondées ${ }^{132}$ ". Si les autorités espagnoles refusent de désavouer officiellement le "visiteur du tabac ", elles font toutefois savoir au consul que des ordres sont secrètement envoyés aux Administrateurs du tabac afin de freiner le zèle de certains de leurs subordonnés ${ }^{133}$.

Il ressort donc de la correspondance consulaire que les maisons de commerce et les navires français retiennent particulièrement l'attention de la Douane de Cadix, ce qui ne saurait étonner étant donnée l'importance du négoce français dans cette place où la contrebande est si générale. Toutefois, il semble, à lire les consuls, que l'administration des douanes ne fasse pas preuve d'autant de zèle quand il s'agit de contrôler les maisons et les bateaux anglais, hollandais ou autrichiens. Aussi y voient-ils la manifestation d'une certaine hostilité aux Français, qui paraît d'ailleurs s'accentuer au fil des années quand, aux visites douanières, s'ajoutent d'autres " vexations " derrière lesquelles se profilent les intérêts du commerce local, de cette bourgeoisie marchande gaditane qui, au cours de ces années, se constitue autour des cargadores et de la corporation des marchands et des boutiquiers - tratantes et tenderos.

Ces " vexations " et les atteintes aux privilèges de la Nation française constituent le dernier thème récurrent de la correspondance consulaire. À en croire les consuls, les plus anciens de ces avantages remontent au traité des Pyrénées de 1659 et tous ont été régulièrement confirmés par les différents Pacte de Famille. Au cours de notre période, ils dénoncent en

130. Arch. nat., Aff. étr. B 276 , lettre de Cassot du 21 décembre 1762.

131. Idem.

132. Arch. nat., Aff. étr. BI 279, lettre de De Puyabry au marquis d'Ossun du 30 janvier 1769.

133. Arch. nat., Aff. étr. B ${ }^{\mathrm{I}} 279$, lettre de De Puyabry du31 octobre 1769. 
particulier les multiples tentatives du négoce local pour éloigner les Français de la Carrera de Indias et pour exercer, par l'intermédiaire de leurs instances représentatives, comme l'Université des chargeurs pour les Indes ou les corporations (Gremios), une pression constante sur la Couronne afin d'obtenir un droit de regard, voire de contrôle, sur les activités des maisons étrangères, au premier rang desquelles, bien sûr, les françaises.

\section{Les tentatives d'ingérence des "Consulats " locaux dans les affaires de la Nation}

Au début de l'année 1764, le Roi d'Espagne ordonne le recensement de "tous les étrangers résidant dans toute l'étendue de ses États". Convoquée le 11 janvier en la maison du consul, l'Assemblée de la Nation française n'y trouve rien à redire dans la mesure où cette opération a pour but " de connaître les négociants étrangers qui commercent en Espagne et d'éviter des abus qui ont souvent lieu envers des particuliers qui se disent étrangers quand cela leur convient et Espagnols quand ils en peuvent tirer avantage ", ce qui serait notamment le cas des Irlandais dont " la plupart sont sous le pavillon anglais en temps de paix et réclament des privilèges espagnols en temps de guerre ${ }^{134}$ ". Versailles ayant donné son accord, les négociants français acceptent donc de se faire inscrire sur les listes du padron ${ }^{135}$.

Trois ans plus tard, un nouveau pas est franchi quand une cédule du 7 juin 1767 leur impose de " conter par pièces authentiques au prieur et consul du Consulat espagnol de Cadix quelles sont les personnes qui composent [leurs] compagnies, celles qui s'y intéressent, avec quels fonds ${ }^{136}$ ". Selon le consul, cette cédule a été obtenue par "le Consulat de Cadix " (l'Université des Chargeurs) qui cherche par ce biais à se faire une idée précise de l'activité des négociants français. Pendant près de dix ans la Nation et les consuls vont combattre cette cédule, tant sur place à Cadix qu'auprès des Cours de Madrid et de Versailles. Leur opiniâtreté paye finalement en mars 1776, lorsque le Roi d'Espagne accepte que les dispositions de cette cédule ne s'appliquent qu'aux seules maisons fondées après le mois d'octobre $1775^{137}$. Toute nouvelle société française se voit donc désormais contrainte de remettre une copie de son contrat d'association au président de la Casa de Contratacion. Désireux de voir se développer les affaires de ses compatriotes autant que le commerce du royaume, le consul De Mongelas juge cette procédure contraire aux intérêts des négociants, car elle pourrait les inciter à dissimuler la réussite de leurs opérations, à ne pas conclure de nouvelles associations ou à ne plus fonder de nouvelles sociétés, pour ne pas avoir à rendre public leurs affaires et risquer par là,

134. Arch. nat., Aff. étr. BI 277, lettre de Cassot du 13 janvier 1764.

135. Arch. nat., Aff. étr. BI 277, lettre de Cassot du 28 février 1764.

136. Arch. nat., Aff. étr. BI 278, lettre de De Puyabry du 11 août 1767.

137. Arch. nat., Aff. étr. $B^{\mathrm{I}} 282 \mathrm{f}^{\circ} 25$, «Traduction et copie de l'ordre de la Cour d'Espagne au Président de la Contractation de Cadix du 19 mars 1776 au sujet de la cédule de 1767 " jointe à la lettre de De Mongelas du 26 mars 1776 . 
le secret étant indispensable à la réussite, de perdre une partie de leur crédit sur les autres places européennes :

\begin{abstract}
"Quelle nécessité y-a-t-il qu'un négociant dévoile aux yeux d'un tribunal les progrès, les fruits de son industrie? Est-il en état lui-même de les évaluer, de les fixer? Ceux qui, par le désir qui reste en eux de s'en retourner dans leur patrie, soustrairont à la curiosité une partie de leurs affaires, seront publiquement reconnus pour négociants peu instruits, peu habiles. Ce silence de leur part affaiblira leur crédit, diminuera la confiance. En un mot, toute opération de commerce doit être à l'abri de toute recherche ${ }^{138}$. "
\end{abstract}

Forte de son premier succès de 1767, l'Université des Chargeurs pour les Indes avait continué son lobbying et obtenu, en octobre 1769, une nouvelle cédule obligeant clairement les négociants français à lui présenter directement leurs contrats de société et à lui déclarer la valeur des fonds avec lesquels ils travaillaient sur la Carrera. Bien entendu, la réaction française ne s'était pas fait attendre et le consul s'était empressé de dénoncer " cette espèce d'inquisition que le Consulta espagnol [voulait] établir envers les étrangers ", tout comme il avait également dénoncé le fait que le "Consulat " soit sorti du cadre de ses attributions dans la mesure où il n'avait été établi que pour " prendre connaissance des affaires qui concern[aient] les Indes " et non celles des étrangers " qui ne [pouvaient] $\mathrm{y}$ avoir aucune relation puisque tout le commerce aux Indes leur [était] interdit ${ }^{139}$ ". C'est certainement parce qu'ils avaient refusé de se soumettre à cette nouvelle cédule, que les négociants français avaient dû subir l'année suivante l'embargo sur les fonds qu'ils faisaient revenir d'Amérique aux noms de leurs épouses et de leurs fils " espagnols".

Cette même année 1770, en octobre, était arrivé à Cadix la nouvelle du remplacement par une Contribution Unique « des droits de millones, d'alcavala et de cent sur la consommation des comestibles et de quelques autres articles ${ }^{140}$ ». Cette fois, la Nation française avait pris les devants et adressé à Madrid une offre d'abonnement sur la base d'un " tableau des fonds " évaluant à 4340000 piastres le total des capitaux détenus par les 72 maisons de commerce en gros et à 4768000 piastres ceux des 32 maisons de détaillants français installés dans la ville ${ }^{141}$. En avril 1772, le Conseil des Finances accepte d'abonner à l'Unique Contribution les seuls " négociants en gros ", mais entend quand même soumettre " les détaillistes et artistes " à déclarer leurs fonds, séparément. Tout en protestant vivement contre cette "division ou séparation que l'on entend faire [d'elle] ", la Nation accepte cependant de se plier aux exigences espagnoles, sur le conseil de l'ambassadeur d'Ossun ${ }^{142}$. Enfin, en mars 1773 , une troisième

138. Arch. nat., Aff. étr. $\mathrm{B}^{\mathrm{I}} 282 \mathrm{f}^{\circ}$ 31, lettre de De Mongelas du 12 avril 1776.

139. Arch. nat., Aff. étr. B 279 , lettre de De Puyabry du 10 octobre 1769.

140. Arch. nat., Aff. étr. BI 279, lettre de De Puyabry du 30 octobre 1771.

141. Arch. nat., Aff. étr. $B^{\mathrm{I}} 279$, lettres de De Puyabry des 22 mars et 23 avril 1771; copie d'une lettre des députés de la Nation au marquis d'Ossun, du 16 avril 1771.

142. Arch. nat., Aff. étr. BI 280, lettres de De Puyabry des 10 et 21 avril 1772. 
cédule oblige « tous les négociants étrangers en gros et en détail de tenir leurs livres et écritures en langue castillane ${ }^{143}$ ".

En moins de dix ans, les autorités espagnoles sont donc parvenues à grignoter quelques-uns des privilèges reconnus aux Français par les traités de commerce. Tout cela n'est pas sans inquiéter le consul De Mongelas qui, à la fin de notre période, fait part de ses craintes à l'idée qu'un nombre croissant de ses compatriotes puissent envisager d'adopter la nationalité espagnole dans le but de poursuivre sans contraintes leurs affaires avec l'Amérique. Cette inquiétude est renforcée par la publication d'un décret de Charles III du 23 avril 1776 qui ramène de vingt à douze le nombre d'années de résidence au terme desquelles un étranger ayant épousé une Espagnole et acquis des biens fonds, peut demander sa naturalisation :

"Nous devons cependant y voir d'un œil inquiet une ordonnance qui peut affaiblir et diminuer le Corps de la Nation française établie à Cadix. Plusieurs, sous l'appât du commerce des Indes qui les a toujours flattés, et par cette réduction à 12 années de séjour en Espagne, pourraient s'en séparer. Peut-être même arriverait-il que les émigrations de France augmenteraient (abus bien essentiel à réformer). Il ne faut point se flatter qu'après une fortune un Français expatrié, déjà naturalisé espagnol, conservât l'idée de retour en France. Les habitudes contractées avec des femmes espagnoles, des familles qui avec le temps deviennent nombreuses font perdre tout souvenir d'un pays qui fut cher, mais qui n'est plus rien en comparaison d'un autre qui offre des liaisons cimentées par le sang des familles ${ }^{144}$."

Son inquiétude augmente dans les semaines qui suivent la publication du décret car il lui semble que plusieurs Français parmi ceux qui sont installés depuis quelques années seulement, et non parmi les familles implantées depuis plusieurs générations et gardant de solides attaches en France, pourraient se laisser tenter et choisir la nationalité espagnole. Bien qu'il s'agisse surtout de " négociants ordinaires " et de gens qui, à ses yeux, font peu d'efforts pour favoriser la navigation et les fabriques françaises, la Nation s'en trouverait affaiblie. Aussi conviendrait-il, selon le Consul, de susciter dans le négoce français un regain d'intérêt pour la nationalité française, par exemple en distinguant certains négociants parmi les plus méritants, à qui le Roi pourrait accorder des lettres de noblesse :

\begin{abstract}
"Beaucoup de nos meilleures maisons de commerce sont conduites par les fils ou parents de personnes qui résident en France et ceux-ci, après quelques années de travail, font place à d'autres et vont à l'aide de leur fortune acheter des charges dans leur province ou y jouir de leur revenu. Mais ceux qui ont un établissement à Cadix, qui y ont contracté des habitudes irréparables, se flattent de trouver les Indes ouvertes et sont sûrs d'augmenter leur bien par la facilité qu'ils ont d'y commercer avec leurs propres enfants. Quelques-uns restent fidèles au royaume. Mais je vois malheureusement quelques riches Français devenus absolument Espagnols et perdus pour leur patrie. En effet, quel autre raisonnement que celui de l'intérêt peut-
\end{abstract}

143. Arch. nat., Aff. étr. B 281, lettre de De Puyabry du 30 mars 1773.

144. Arch. nat., Aff. étr. $B^{\mathrm{I}} 282$ f ${ }^{\circ}$ 45, lettre de De Mongelas du 28 mai 1776. 
on attendre d'un homme souvent sans aucun principe d'éducation qui, parvenu à une fortune considérable, a formé des habitudes dans le lieu de son opulence dont les mœurs sont chères à son caractère, qui à peine connaît ceux de son pays natal [...]. Je crois que rien ne serait plus fait pour conserver nos négociants attachés au pavillon que des récompenses telles que des lettres de noblesse. Celles accordées au sieur Sahuc n'ont pas fait grande sensation. Ses mérites n'ont rien d'éclatant aux yeux de la Nation. Peu de gens sont susceptibles de cette faveur ${ }^{145}$."

C'est certainement pour essayer de renforcer ces liens avec la mèrepatrie que le consul propose en janvier 1778, à l'occasion de l'Assemblée de la Nation qui procède à l'élection des députés, les noms de négociants " mariés à des filles de Français nées en Espagne " et qu'il estime être " les plus propres et les plus capables de veiller et de penser au bien du commerce ". Mais les membres de la Nation lui opposent une fin de non-recevoir en objectant de l'article XIX du règlement de 1728 qui empêche " les Français naturels ou naturalisés qui auront épousé des femmes nées espagnoles sans la permission du roi " d'être élus députés, tout autant que les fils nés de ces unions. Le conservatisme de la Nation désole alors De Mongelas qui ne cache pas au ministre à quel point il trouve " dangereux de tolérer que les enfants mâles qui naissent en Espagne de pères français soient exclus du droit d'assister aux assemblées et inhabiles à posséder les charges de la Nation " car, explique-t-il, cela réfrène leurs " sentiments patriotiques " et les incite alors à se faire naturaliser Espagnols, naturalisation qui, de surcroît, leur ouvre pleinement et sans contraintes la porte du commerce des Indes ${ }^{146}$.

Au moment où se termine notre correspondance, les maisons françaises de Cadix doivent faire face à une dernière forme de " vexation " : les visites de l'Inquisition. Elles commencent en 1777 et, dans une lettre chiffrée, De Mongelas fait part de son inquiétude devant ces perquisitions impromptues qui ne respectent pas les privilèges juridiques des étrangers et qui portent atteinte à l'honneur et à la réputation de ceux qui en font l'objet :

"Le tribunal de l'Inquisition renouvelle plus fortement que jamais ses recherches sur les livres, propos, tableaux et généralement sur tout ce qui a trait à la religion. Il fait des visites dans toutes les maisons, au milieu même de la nuit. Nos nationaux en ont essuyé quelques unes qui ont jeté la crainte parmi eux. L'Inquisiteur m'avait cependant promis de n'en rien faire; malgré cela ces visites redoublent [...]. Ces visites sont dangereuses pour des maisons étrangères par le scandale qu'elles occasionnent dans un pays où le préjugé guide la plupart des hommes, et elles sont vexatoires par l'autorité absolue qu'a ce tribunal de descendre chez un particulier quand bon lui semble et sans aucune intervention du juge militaire ${ }^{147}$."

Le consul se dit choqué par les méthodes utilisées et par le comportement des inquisiteurs lorsqu'ils visitent les demeures de ses compatriotes,

145. Arch. nat., Aff. étr. $B^{\mathrm{I}} 282$ f 58 , lettre de De Mongelas du 12 juillet 1776.

146. Arch. nat., Aff. étr. Bl 284, lettre de De Mongelas du 20 janvier 1778.

147. Arch. nat., Aff. étr. B' 283, extrait chiffré d'une lettre de De Mongelas du 4 février 1777. 
au nombre desquels on peut supposer qu'il y ait des protestants. Ils vident les bibliothèques, confisquent certains tableaux et marquent systématiquement à la craie les façades des maisons qu'ils ont visitées, ce qui à l'en croire pourrait détourner de ces boutiques une partie de leur clientèle habituelle :

"Chaque jour ce tribunal fait les recherches les plus inouïes. Il fait exactement le tour de toutes les maisons et j'ai la douleur de voir que les Français sont les seuls étrangers qu'il ose visiter impunément. On va distraire le négociant occupé de son commerce et le forcer, le crucifix à la main, d'ouvrir sa bibliothèque, ses coffres, ses armoires. Dernièrement un de nos orfèvres fut enlevé par un inquisiteur et conduit à sa maison de campagne pour y subir une visite. On fait même plus. On descend dans d'autres maisons quand les propriétaires sont absents, et cela pour enlever une peinture qui représente une tête de femme. Tous nos nationaux tremblent à l'aspect d'une force si majeure [...]. Depuis quatre jours toutes les maisons sont marquées avec de la craie. Il semble que la sûreté publique, que l'asile de chaque individu soit devenu la proie du pouvoir arbitraire et invisible. Nos nationaux semblent être l'objet [des vexations que commet ce tribunal] cependant que nul propos impie ne sort de leurs bouches. Leurs actions sont honnêtes. Un homme peut être cité devant le clergé pour quelques propos indécents ou actions malhonnêtes, mais que sa maison soit à l'abri de toute recherche inique qui emporte toujours avec elle une publicité et un scandale qui n'est que trop dangereux parmi un peuple fanatique à l'excès où l'introduction des livres est défendue ${ }^{148}$."

Le 10 mai 1778, une des principales maisons françaises de la ville, Cayla Solier Cabane \& Jugla, est perquisitionnée. Plusieurs tableaux appartenant à Jugla sont embarqués ainsi que 86 volumes de la bibliothèque " dont la plupart sont leurs livres de dévotions, et entre autres celui des psaumes, l'Ancien Testament et les sermons de M. Sauriri ${ }^{149}$ ". Le lendemain et le surlendemain, deux autres visites ont lieu. Cette fois, 90 ouvrages supplémentaires sont soustraits de leur bibliothèque par le commissaire du SaintOffice, ainsi que plusieurs autres tableaux ${ }^{150}$. Une quatrième perquisition a lieu le 18 mai et l'Inquisition emporte 28 nouveaux livres ${ }^{151}$. Quelques jours auparavant, un autre Français, Durays, s'était lui vu confisquer pas moins de 200 ouvrages ${ }^{152}$.

Bien que, selon le Consul, ces perquisitions soient contraires à l'article 28 du traité d'Utrecht de 1714, qui défend que les Français soient inquiétés en raison de leurs convictions ${ }^{153}$, l'émotion provoquée par cette affaire est réelle dans la communauté française. Pour calmer la Nation, l'Inquisiteur doit alors assurer le consul qu'à l'avenir il procèdera à ces visites avec plus de discrétion, avec " tous les ménagements, douceur, l'honnêteté et les égards possibles ", et qu'il ne remettra pas au tribunal de Séville les livres

148. Arch. nat., Aff. étr. BI 283, extrait chiffré d'une lettre de De Mongelas du 21 mars 1777. 149. Arch. nat., Aff. étr. B 284 , lettre de Poirel du 12 mai 1778.

150. Idem.

151. Arch. nat., Aff. étr. B 284 , lettre de Poirel du 19 mai 1778.

152. Arch. nat., Aff. étr. B 284 , lettre de Poirel du 12 mai 1778.

153. Idem. 
et les tableaux confisqués chez les Français mais les conservera en dépôt " dans une caisse avec une marque particulière " en attendant que leurs propriétaires obtiennent de la Cour le droit de les récupérer ${ }^{154}$.

La correspondance des consuls de France telle qu'elle a été conservée dans la sous-série $\mathrm{B}^{\mathrm{I}}$ du fonds des Affaires étrangères nous permet donc d'avoir une vue d'ensemble sur la nature des activités des négociants français installés à Cadix au cours de ces quinze dernières années du régime de la Carrera de Indias. Leurs affaires, qui reposent essentiellement sur le commerce des toiles et des étoffes, sur le négoce des piastres et sur le prêt à la grosse aventure, semblent bien se porter pendant cette période et être dans une phase ascendante, malgré une petite crise perceptible vers 1772 , quand elle emporte une des plus importantes maisons de la ville, Béhic Tavenot \& $\mathrm{C}^{\mathrm{ie}}$, victime de " malheurs imprévus et réitérés ${ }^{155}$ ", et qui laisse un passif de " un million et trois cent tant de mille piastres ${ }^{156}$ ". Alors que la monarchie espagnole cherche à remplacer progressivement le monopole par un système plus libre en ouvrant, à partir de 1765, le commerce avec l'Amérique à d'autres ports, les consuls formulent davantage de plaintes contre l'Administration des douanes ou le Consulat des Indes - mais c'est là leur fonction - qu'ils ne se réjouissent des opportunités offertes à leurs compatriotes ou ne vantent les réussites de certains d'entre eux. On rappelle inlassablement ce qui ne va pas ou risque de ne plus aller, mais on tait ce qui réussit - ou alors on ne l'exprime qu'à travers le chiffrage. En filigrane, on peut également saisir quelques informations sur la composition de la Nation et sur son fonctionnement, notamment à travers les copies des procès-verbaux de ses assemblées qui sont réunies régulièrement, au moins une fois par an, parfois plus, afin d'élire les deux députés, élections pour lesquelles la participation ne paraît d'ailleurs pas massive au vu des longues listes d'absents, excusés ou non.

Cette correspondance ne saurait toutefois être considérée comme suffisante pour percevoir dans leur globalité la vie et les activités des Français de Cadix. Il faudrait certainement la compléter, par exemple par celle qui est conservée dans le fonds Marine $\mathrm{B}^{7157}$ qui, à moins de faire doublon, pourrait contenir des pièces complémentaires comme ces mémoires ou ces états du commerce adressés au ministre de la Marine par les députés de la Nation et dont les consuls parlent dans leurs lettres. Nous tâcherons de les parcourir prochainement. Quant à la communauté des Français établis dans la ville, elle peut être mieux approchée à travers l'exploration des actes de chancellerie ${ }^{158}$, lesquels nous révèlent, à côté des négociants et

154. Arch. nat., Aff. étr. BI 284, lettre de Poirel du 15 mai 1778.

155. Arch. nat., Aff. étr. B 280 , lettre de De Puyabry du 24 janvier 1772.

156. Arch. nat., Aff. étr. $B^{\mathrm{I}} 280$, lettre de De Puyabry du 10 mars 1772.

157. Arch. nat., Mar. B7, correspondance à l'arrivée avec documents et mémoires sur le commerce; B7 421 à B7 439 Espagne, Italie, Nord.

158. Archives diplomatiques de Nantes, Consulat de Cadix, Registres des actes de chancellerie, registres 211 à 243, décembre 1703 à mai 1793. 
des boutiquiers classés dans le Corps de la Nation, toute une foule e petites gens, très souvent d'origine limousine, et qui exercent les métiers d'aubergiste, de cuisinier, de boulanger, de perruquier, de porteur d'eau, etc. Dans la mesure où les capitaines français étaient tenus de faire leur déclaration auprès du Consulat dès leur arrivée à Cadix, ces actes de chancellerie nous renseignent aussi sur les mouvements de navires en provenance de France et permettraient certainement d'approcher d'un peu plus près cet aspect du grand cabotage européen.

À notre connaissance, aucun dépouillement systématique de la correspondance des consuls de Cadix n'aurait été faite. Il est vrai que la tâche est immense puisque la sous-série $B^{\mathrm{I}}$ comprend 75 registres qui englobent une période allant de 1666 à 1779. Il pourrait toutefois être intéressant, à la lumière de cette première synthèse, de parcourir ceux des années 1720 et 1740, ne serait ce que pour se faire une idée d'ensemble sur la façon dont les premières réformes bourboniennes en matière de commerce colonial - initiées par le Projet pour les galions et les flottes d'avril 1720 - ont été ressenties par la Nation française et par ceux qui la représentaient alors. 


\section{RÉSUMÉ}

Dans la seconde moitié du XVIII ${ }^{\mathrm{e}}$ siècle, la Nation française de Cadix, forte de plus d'un millier de membres, se pose comme la première communauté française d'Espagne. Porte d'entrée de l'immense empire espagnol d'Amérique, la cité andalouse est depuis le $\mathrm{xvI}^{\mathrm{e}}$ siècle l'un des principaux ports de la façade atlantique de l'Europe. Les Français de Cadix sont placés sous la protection d'un consul, représentant du Roi de France, chargé à la fois de défendre les intérêts de ses compatriotes et d'informer le ministre de la Marine de tout ce qui se passe dans la ville et dans le port. Aussi la correspondance de ce consul nous permet-elle de saisir l'ampleur, la nature et les méthodes du commerce français et de comprendre comment les négociants français s'immiscent alors dans le commerce d'Amérique en contournant le monopole de la Casa de Contratacion, notamment pour tirer profit du fructueux trafic des piastres d'argent. Bien intégrés dans le négoce gaditan, les marchands français doivent cependant faire face aux revendications croissantes de leur confrères espagnols qui tentent par diverses manœuvres auprès des autorités locales, à la faveur des réformes de Charles III et de la libéralisation de 1778 , de reprendre la main pour éviter que la manne américaine ne leur échappe. Des capacités du consul à défendre les privilèges autrefois concédés par le Roi Catholique et les intérêts généraux du commerce d'Espagne dépendra donc le devenir des négociants français de Cadix.

\section{ABSTRACT}

In the second half of the eighteenth century, more than one thousand French people lived in Cadiz. They represented the most important French community in Spain. Since the sixteenth century, the Andalusian city had been one of the main Western European ports. In Cadiz, French people were living under the protection of a consul, who represented the French King and who was in charge of defending his fellow countrymen's interests. Moreover the consul had to inform the Navy Minister of everything which was happening in the city. That's why the consul's letters help us understand what the French trade relly consisted in and how important it was. French merchants traded with the Sapnish colonies in America, in spite of the Casa de Contratacion's monopoly, hoping to profit from the silver piastres' trafic. Althoughthey were perfectly inbtegrated in the society of Cadiz merchants, French merchant had to face the growing protests of their Spanish counterparts who tried to take advantage of Charles III's reforms in order to gain control of the colonial trade with America. 